\title{
A Sampling Probe for Fluctuating Concentration Measurements in Supersonic Flow
}

\author{
By \\ Olivier Christian Xillo \\ Thesis Submitted to the Faculty of the \\ Virginia Polytechnic Institute and State University \\ In partial fulfillment of the requirements for the degree of
}

MASTER OF SCIENCE

IN

AEROSPACE ENGINEERING

APPROVED:

Dr. Joseph A. Schetz, Chairman

Dr. Wing F. Ng

Dr. Roger L. Simpson

April 24, 1998

Blacksburg, Virginia

Key Words: Fluctuating, Supersonic, Concentration, Measurements 


\title{
A Sampling Probe for Fluctuating Concentration Measurements in Supersonic Flow
}

\author{
By \\ Olivier Christian Xillo \\ Aerospace and Ocean Engineering
}

(ABSTRACT)

The study of mixing processes in supersonic flow has a great interest for a number of applications including scramjet developments. However, the usual tools employed are very limited. Sampling probes generally have a poor frequency response, and that makes measurements of fluctuating concentration problematic. The goal of this study is to design, build and test a probe capable of measuring concentration fluctuations of at least $1 \mathrm{kHz}$. This study shows the design of such a probe and how a high frequency response was achieved. A prototype was built and tested. The tests consist of a calibration phase and experiments inside a hypersonic wind tunnel. These last tests served two purposes. The first one was to verify that the measurements made with the probe gave the correct value of the concentration. The second purpose was to assess the frequency response of the probe by putting it through a concentration step change. Tests were conducted with various known mixtures of Helium and air from 0\% to 100\% flowing at Mach 2.0 with $T_{t}=300{ }^{\circ} \mathrm{K}$ and $P_{t}=4.4 \mathrm{~atm}$. The probe proved capable of measuring concentration fluctuations up to approximately $2.5 \mathrm{kHz}$ while also giving the adequate accuracy for values of mean concentration. 
I would first like to sincerely thank my advisor, Dr. Joseph SCHETZ. He made it possible for me to accomplish my military service for the French government in his laboratory and, meanwhile, to get a master's degree. That was a terrific opportunity and a great experience. I want to thank him for his understanding and help with my special situation. I also want to thank him for his precious guidance and help during this study. I also want to thank Dr. Wing NG. His expertise in aspirating sampling probes was really helpful and appreciated. I also thank Dr. Raymond FULLER at Wright Laboratory whose previous experience with concentration probe was of great help. Finally, I want to thank Dr. Roger SIMPSON for serving as a member on my committee.

I also appreciated the help of Mrs. Carlene ARTHUR, Betty WILLIAMS and Wanda FOUSHEE. They helped me with all the administrative tasks, which can sometimes be confusing for a foreign student. I also want to pay homage to the patience and competence of the technicians in the machine shop, especially Mr. Bruce STANGER. I guess that some of my tiny designs gave him nightmares. The help of Mr. Gary STAFFORD was also greatly appreciated. Finally, I had a great time working with my labmates Lance JACOBSEN and Diane WILSON.

Last but not least, I would like to thank my family and friends for their support during the long and arduous process of completing this work. 


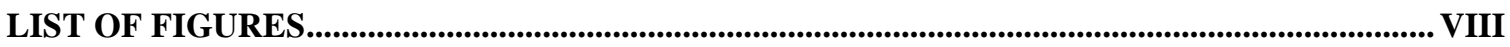

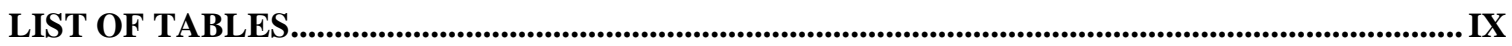

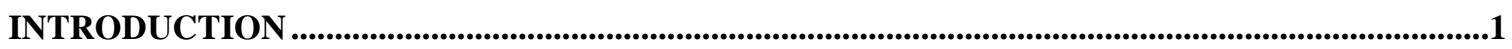

PRINCIPLE OF OPERATION .......................................................................................................................3

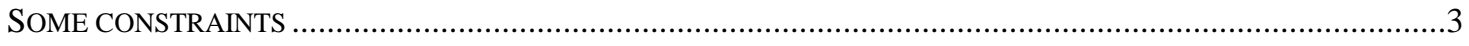

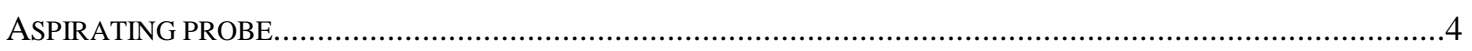

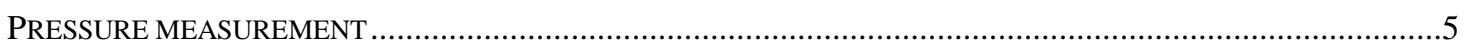

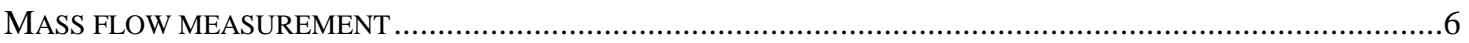

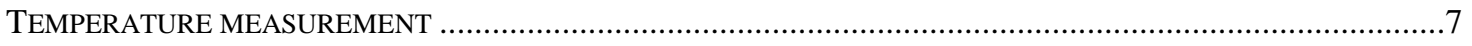

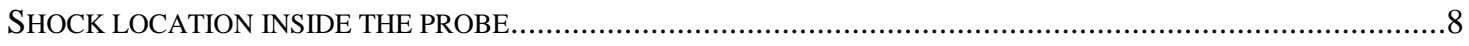

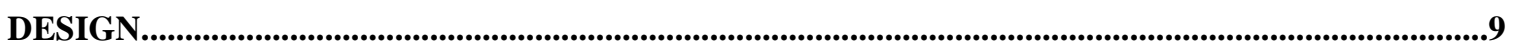

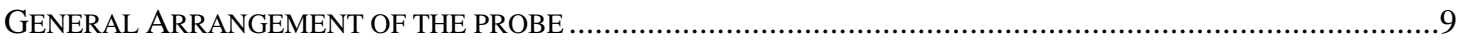

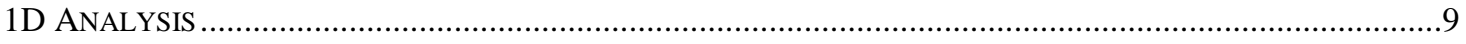

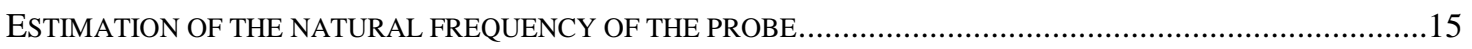

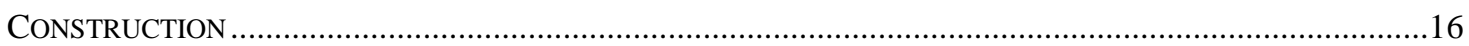

DATA ACQUISITION AND CALIBRATION ...............................................................................................20

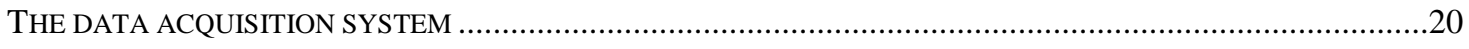

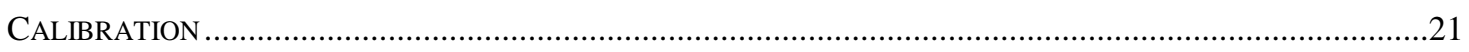

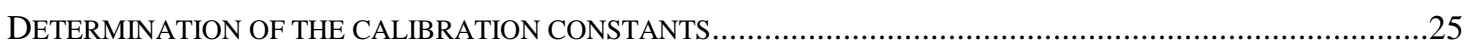

EXPERIMENTS.......................................................................................................................................29

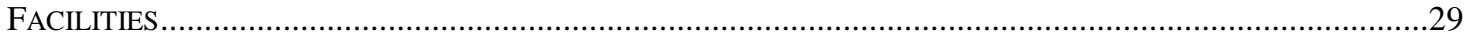

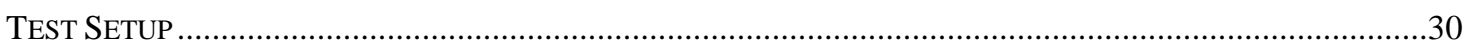

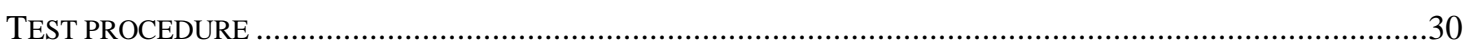

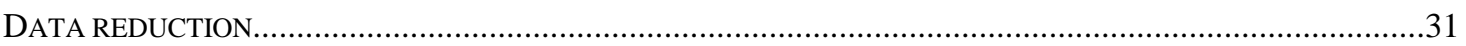

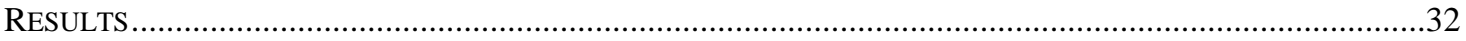




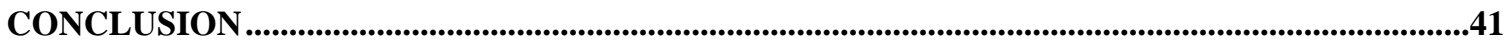

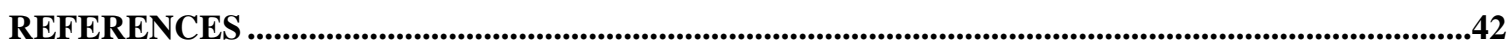

APPENDIX A ..........................................................................................................................................................44

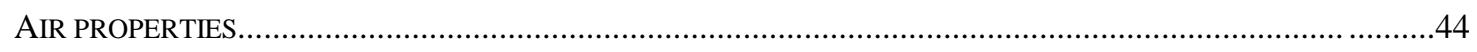

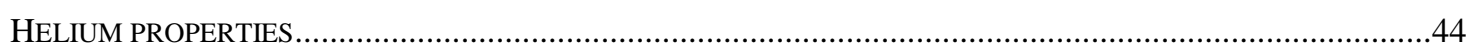

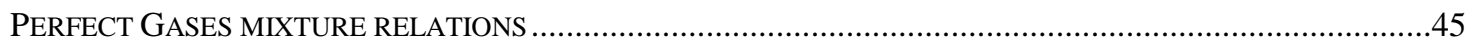

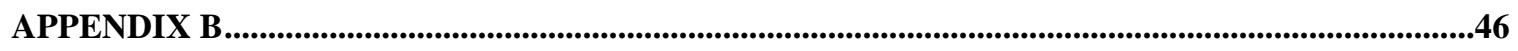

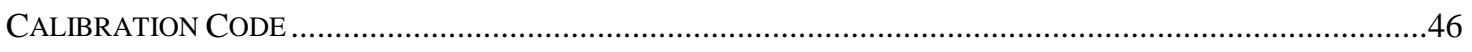

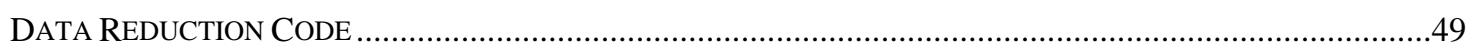

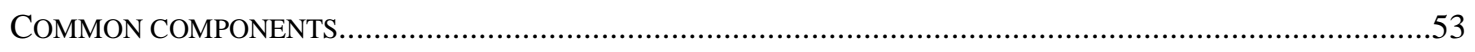

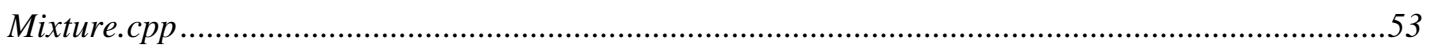

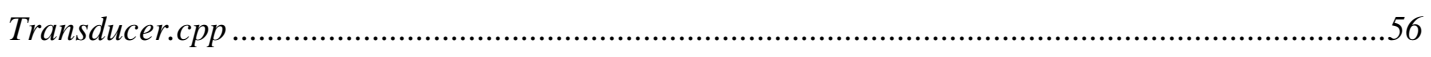

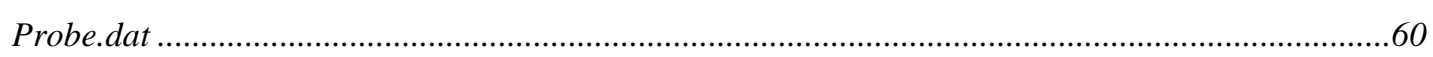

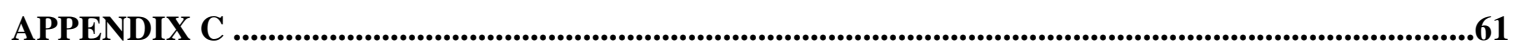

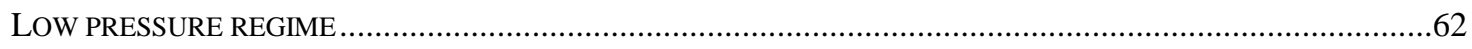

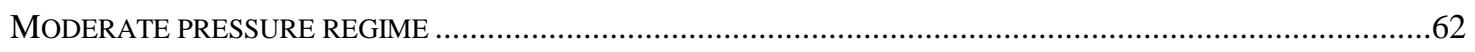

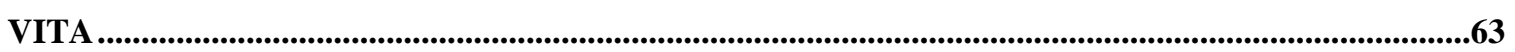




\begin{tabular}{ll}
$X$ & helium mole fraction \\
$R$ & specific gas constant \\
$\gamma$ & ratio of specific heats \\
$M$ & Mach number \\
$\rho$ & density \\
$U$ & velocity \\
$A$ & cross-sectional area \\
$P_{t}$ & total pressure \\
$T_{t}$ & stagnation temperature \\
$T_{e}$ & recovery temperature \\
$T_{w}$ & hot-film temperature \\
$V$ & hot-film voltage \\
$R_{w}$ & sensor operating resistance \\
$R_{s}$ & bridge resistance \\
$N u$ & Nusselt number \\
$\operatorname{Re}$ & Reynolds number based on the diameter of the hot-films \\
$d$ & hot-film diameter \\
$l$ & active length of the hot-film \\
$k$ & thermal conductivity \\
$\mu$ & viscosity \\
$a, b, m$ & calibration constants \\
$F(s)$ & Fourier transform of s \\
\hline
\end{tabular}

Subscripts

None mixture property 


$\begin{array}{ll}\text { Air } & \text { air property } \\ \text { He } & \text { helium property } \\ \text { in } & \text { inlet property } \\ h f & \text { sensor plane property } \\ s h^{-} & \text {shock upstream property } \\ s h^{+} & \text {shock downstream property } \\ t h & \text { throat property }\end{array}$


Figure 1 - Aspirating probe by Ninneman and $\mathrm{Ng}(1992)$.............................................. 1

Figure 2 - Shock wave in front of the probe ................................................................. 3

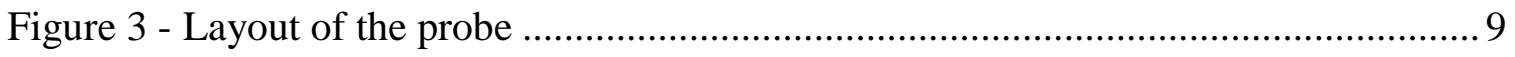

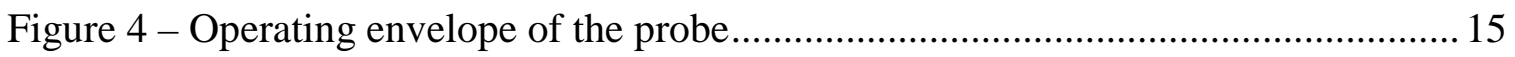

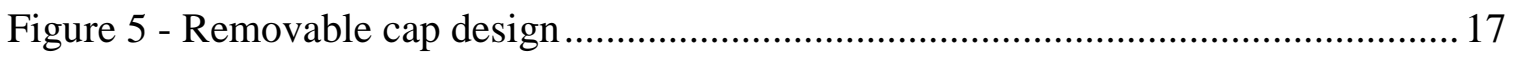

Figure 6 - Possible geometries of the throat insert..................................................... 18

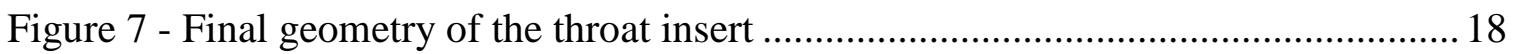

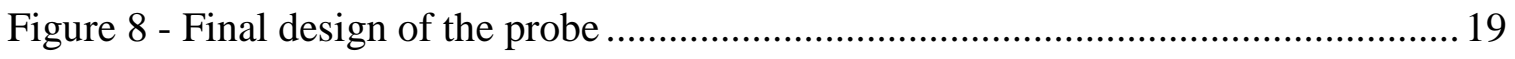

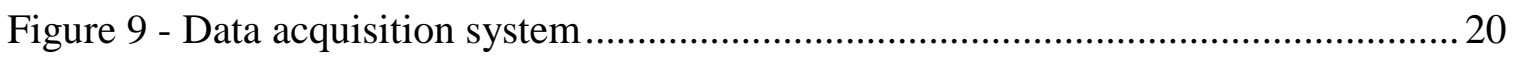

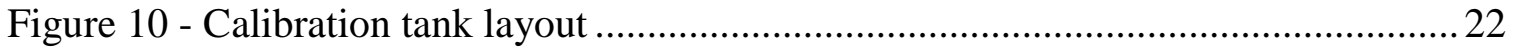

Figure 11 - Typical calibration curves for a given concentration ..................................23

Figure 12 - Calibration curves (low overheat hot-film) ............................................ 24

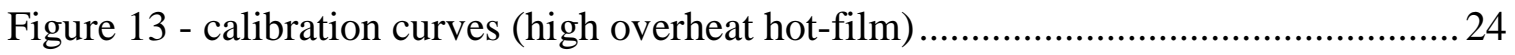

Figure 14 - Hypersonic Wind Tunnel Layout .......................................................... 29

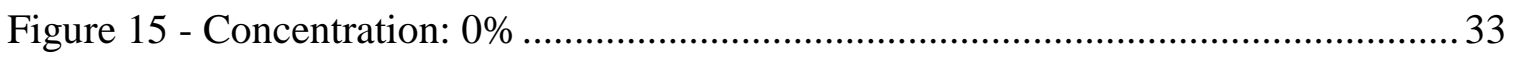

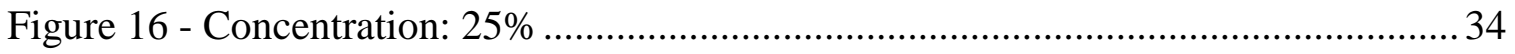

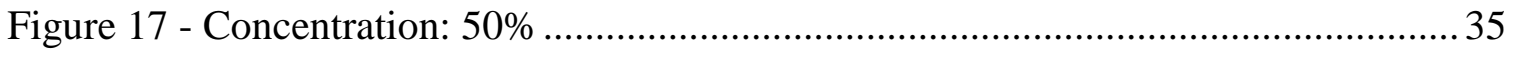

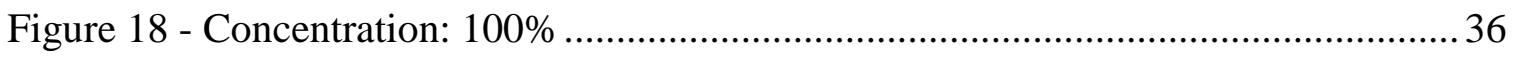

Figure 19 - Concentration signal and idealized step change ........................................ 38

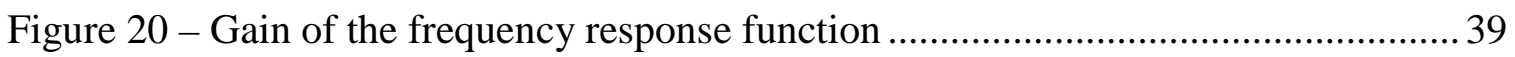

Figure 21 - Gain of the frequency response function (averaged) .................................. 39 


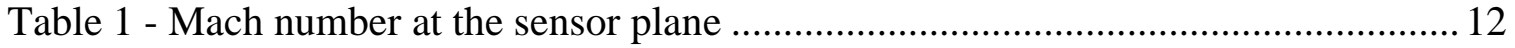

Table 2 - Pressure ratio across the shock and Mach number upstream of the shock ........ 13

Table 3 - Position of the shock and distance to the sensor plane .................................. 14

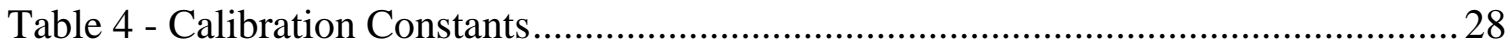


The major aerospace companies are currently studying the design of hypersonic planes for passenger transport. This project involves solving a lot of problems concerning the structure, the materials and the propulsion system to be employed. The actual foreseen propulsion plants are scramjet (supersonic combustion ramjet) engines. The problem in designing such engines lies on the fact that the flow inside the engine is supersonic. With such high-speed flow, the injectant (or combustible) is convected far away from the injector location before the combustion can effectively occur. The key is to control how and where this combustion takes place. Few data are available and we need to learn about what is happening inside these engines, especially how the mixing of the combustible and the airflow operates.

There are several ways to determine the composition of a binary mixture. Some imply sampling a small amount of gas and analyzing it outside the tunnel. Other techniques feature in situ sampling. Ninneman and $\mathrm{Ng}$ (1992) developed an aspirating probe with a sonic throat for use in supersonic flows. A drawing is shown in Figure 1.

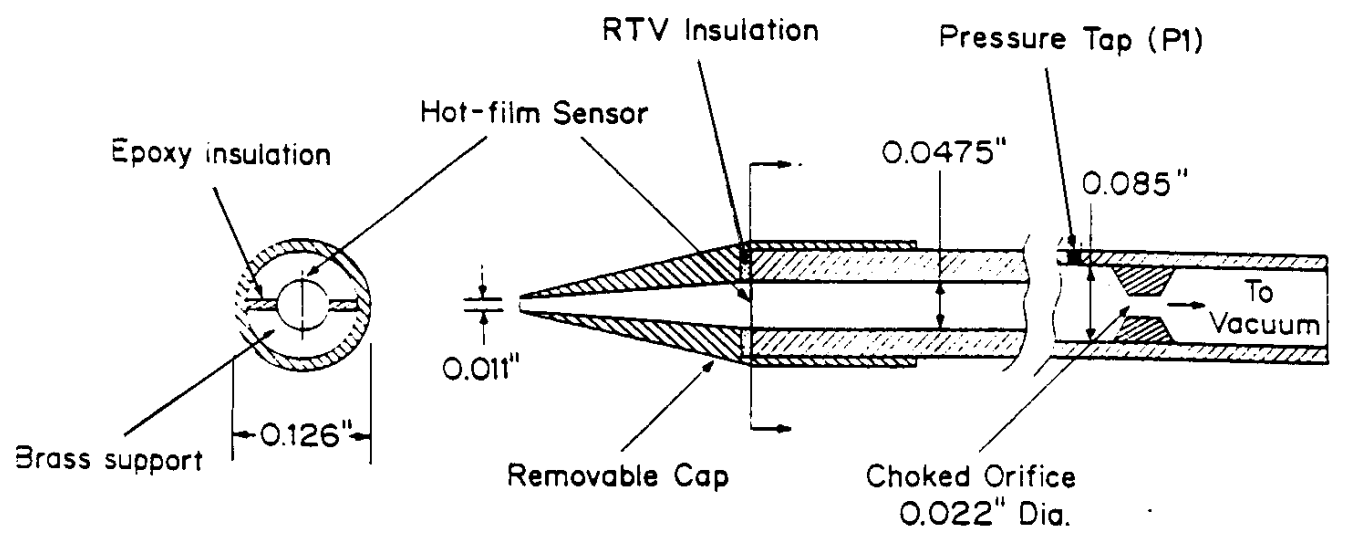

Figure 1 - Aspirating probe by Ninneman and Ng (1992)

Unfortunately, these probes have a poor frequency response $(<200 \mathrm{~Hz})$ and are not suitable to measure fluctuating concentrations. Since mixing involves a lot of turbulent 
phenomena, it seemed natural to develop a probe with a better frequency response: at least $1 \mathrm{kHz}$ was the stated goal. Measurements of concentration fluctuations are critical to improved understanding of turbulent mixing flows and also to the development of turbulence models for use in CFD codes to predict these flows. 


\section{Some constraints}

Determining the composition of a mixture by sampling is not an easy task. Such devices are often bulky and never designed to be used inside a small wind tunnel. Consequently, gas analyzers are usually located outside the tunnel, and samples taken in the tunnel test section are conveyed to the analyzer via a set of tubing. This kind of system has a very low frequency response and is rejected from our scope. Our interest will focus on systems that allow in situ sampling.

Among these devices, one should be careful about the disturbances they introduce in the flow. In subsonic flows, one must sample at the same rate as the local stream velocity; this is called "isokinetic" sampling. Smaller is often the better, but, since we will be working in supersonic flows, we are also concerned by the shock that will develop at the tip of the probe. A detached shock will ruin the measurements, because flow will spill around the tip and species separation can occur. Thus, we want the shock to be attached.
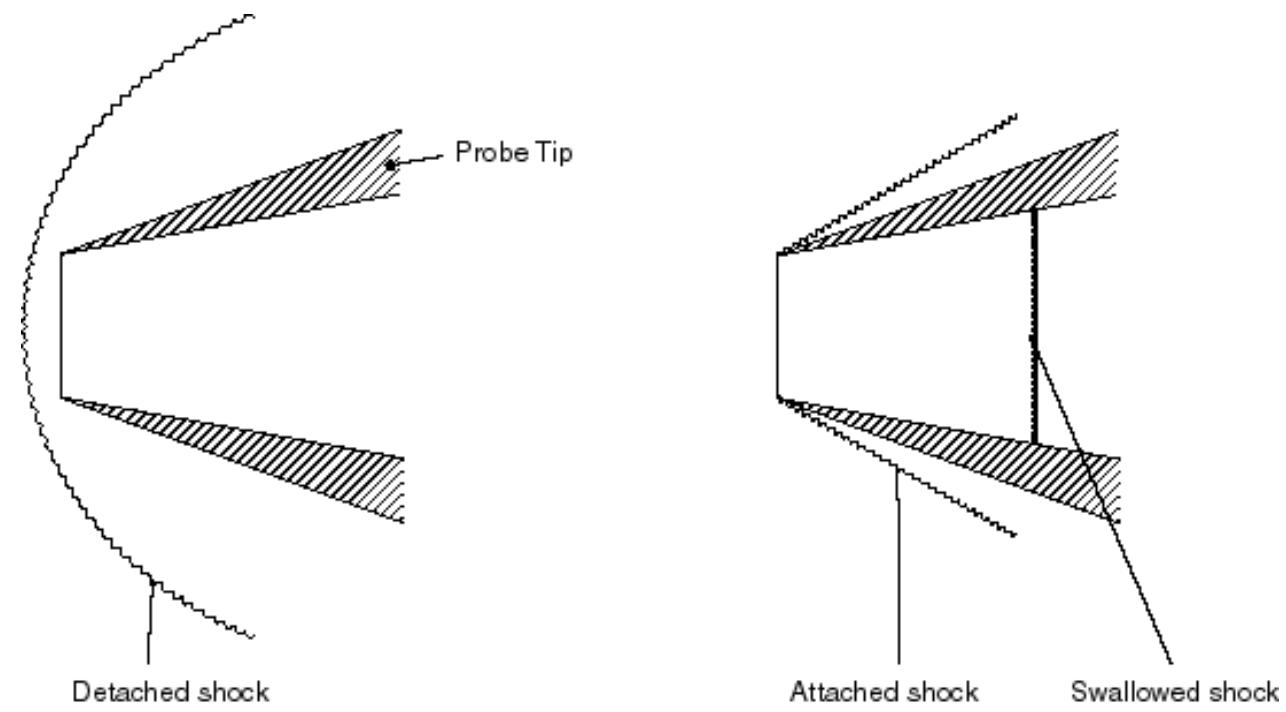

Figure 2 - Shock wave in front of the probe 
Unfortunately, there are no devices that satisfy these constraints and are also capable of determining the composition of arbitrary mixtures of several gases. However, we are more specifically interested in studying binary mixtures (Air/Hydrogen ideally but safety concerns recommend Air/Helium instead). For this binary type of mixtures, solutions exist and involve an aspirating probe like the one developed by Ninneman and $\mathrm{Ng}(1992)$.

\section{Aspirating probe}

Aspirating probes do not determine directly the concentration - or composition - of a binary mixture. The gas is sucked inside a channel inside the probe where several properties of the flow are measured (pressure, temperature...), and by combining these data, it is possible to retrieve the concentration.

The choice of the two gases must be appropriate, since the probe uses the fact that the molecular weight and the physical properties of the two gases are very different. In fact, the greater the difference in molecular weight, the better the sensitivity of the probe.

For the same inlet conditions, the flow properties inside the probe will depend on the composition of the mixture. For any mixture, we can write the steady mass flow equation at two different sections:

$$
\begin{aligned}
(\rho U)_{1} A_{1} & =(\rho U)_{2} A_{2} \\
& =\frac{P_{t_{2}}}{\sqrt{T_{t_{2}}}} \sqrt{\frac{\gamma_{2}}{R_{2}}} A_{2} M_{2}\left(1+\frac{\gamma_{2}-1}{2} M_{2}^{2}\right)^{-\frac{1+\gamma_{2}}{2\left(\gamma_{2}-1\right)}}
\end{aligned}
$$

\section{(Eq. 1 )}

$R_{2}$ and $\gamma_{2}$ depend on the concentration $X$ and the physical properties of each gas. They can be evaluated using the perfect gas mixture relations. 
Consequently, the mass flow equation can be written in the form:

$$
(\rho U)_{1}=\frac{A_{2}}{A_{1}} g\left(P_{t_{2}}, T_{t_{2}}, M_{2}, X\right)
$$

(Eq. 2)

Finding the concentration $X$ involves solving the above equation knowing the values of $(\rho U)_{1}, P_{t_{2}}, T_{t_{2}}$ and $M_{2}$. One key feature of aspirating probe is the incorporation of a throat in the internal channel. At the throat, the Mach number will always be unity if there is sufficient aspiration (i.e. a sufficient pressure difference between the pressure in front of the throat and the backpressure). Thus, the equation becomes

$$
(\rho U)_{1}=\frac{A_{2}}{A_{1}} g\left(P_{t_{2}}, T_{t_{2}}, 1, X\right)
$$

(Eq. 3)

and we only have three quantities to measure:

1. The pressure, $P_{t_{2}}$

2. The mass flow, $(\rho U)_{1}$

3. The temperature, $T_{t_{2}}$

\section{Pressure measurement}

Our goal to achieve a high frequency response led us to reconsider the solution adopted by $\mathrm{Ng}$ and Ninneman for pressure measurements. The use of hypodermic tubing to convey the pressure changes from the pressure tap to the pressure transducer can be very helpful in reducing the size of the probe. Unfortunately, it alters the frequency response. Of course, the connecting tubes could be filled with grease to obtain a better frequency response, but these solutions can become very complex and hard to realize. We preferred 
a simpler solution that consists of putting the pressure transducer inside the probe. By reducing the length of connecting lines, we increase the frequency response. However, this solution requires a miniature pressure transducer with a high frequency response. Such transducers are available from Kulite, Inc. The laboratory had some Kulite XCQ062 from previous experiments. These transducers are only $1.6 \mathrm{~mm}$ in diameter and 5 $\mathrm{mm}$ in length. They have a very high frequency response (about $50 \mathrm{kHz}$ ) and a good precision. Therefore, we decided to use one within the probe.

\section{Mass flow measurement}

There are several ways to measure mass flow, but thermal anemometry seems to be the one that is the most adapted to our needs. The sensor element can be very small, and the frequency response is excellent. There are two types of thermal anemometers: constant current anemometers (CCA) and constant temperature anemometers (CTA). They both have advantages in supersonic flows (Bestion et al. (1983)). However, the availability of constant temperature anemometers in the laboratory made us choose the constant temperature solution. The principle of constant temperature anemometers is to control the voltage applied to a sensor so that its temperature stays constant. When the flow over the sensor changes, the cooling of the sensor changes thus its temperature changes and, consequently, the anemometer reacts by adjusting the voltage. The governing law may be written for supersonic flows:

$$
V^{2}=\frac{\left(R_{s}+R_{w}\right)^{2}}{R_{w}} \pi l k N u\left(T_{w}-T_{e}\right)
$$

(Eq. 4)

The dependence of the voltage on the mass flow is hidden in the Nusselt number. The Nusselt number can be expressed as a function of the Reynolds number, which is proportional to the mass flow. The relation $N u=a \sqrt{\mathrm{Re}}+b$ (Eq. 5) is commonly accepted for air. However, it was found that for Air-Helium mixtures the relation 
$N u=a \operatorname{Re}^{m}$ (Eq. 5a) was more appropriate (Devillers and Diep (1973)). $a$ and $m$ are experimentally determined constants that vary with the concentration of the mixture.

Thermal anemometers use two types of sensors: hot-wires and hot-films. Hot-wires can be very small ( 4 microns in diameter and $1 \mathrm{~mm}$ in length) and have an excellent frequency response. Hot-films are bigger (25 microns) and suffer from a lower frequency response. However, their design makes them less fragile, especially to particle impacts. They can also sustain higher constraints. This last point is important, since we want to use the sensors in supersonic flow, upstream of the shock inside the probe. Another argument that influenced our choice is the reliability of the probe. Even though it is possible to repair the probe, it remains a difficult and delicate task that we want to avoid. Consequently, hot-films offered the best compromise.

\section{Temperature measurement}

Although it may seem one of the easiest measurements to do, it is difficult to get fast, accurate temperature data. The most commonly used device is the thermocouple. It can be very small and very versatile. Unfortunately, the frequency response even for very small wires is below our needs (up to $600 \mathrm{~Hz}$ ).

It is also possible to retrieve the temperature from two hot-films (or hot-wires) operating at different temperatures. The frequency response is then determined by the frequency response of the films and consequently matches our needs. Moreover, since we are already using one hot-film for mass flow measurements, we just need an extra film. We opted for a hot-film probe with two identical parallel sensors. When mounted inside the concentration probe, the two sensors will see the same flow. Remember that we are working in supersonic flows. As a consequence, the hot-films are not sensitive to total temperature but are measuring a recovery temperature $T_{e}$. 
From the governing equations of the hot-films:

$$
\begin{gathered}
V_{1}^{2}=\frac{\left(R_{s_{1}}+R_{w_{1}}\right)^{2}}{R_{w_{1}}} \pi l k N u\left(T_{w_{1}}-T_{e}\right) \\
V_{2}^{2}=\frac{\left(R_{s_{2}}+R_{w}\right)^{2}}{R_{w_{2}}} \pi l k N u\left(T_{w_{2}}-T_{e}\right)
\end{gathered}
$$

\section{(Eq. 6)}

we can easily isolate the recovery temperature and the Nusselt number (TSI IFA 100 Manual)

$$
\begin{gathered}
T_{e}=\frac{\mathrm{A}_{2} V_{1}^{2} T_{w_{2}}-\mathrm{A}_{1} V_{2}^{2} T_{w_{1}}}{\mathrm{~A}_{2} V_{1}^{2}-\mathrm{A}_{1} V_{2}^{2}} \\
N u=\frac{\mathrm{A}_{2} V_{1}^{2}-\mathrm{A}_{1} V_{2}^{2}}{\mathrm{~A}_{1} \mathrm{~A}_{2}\left(T_{w_{1}}-T_{w_{2}}\right)}
\end{gathered}
$$

(Eq. 7)

where $\mathrm{A}_{i}$ is defined as $\mathrm{A}_{i}=\frac{\left(R_{s_{i}}+R_{w_{i}}\right)^{2}}{R_{w_{i}}} \pi l k$

\section{Shock location inside the probe}

Since the sampled flow is supersonic and the flow through the throat in the probe is sonic, there must be a shock somewhere inside the probe. In the original design, the internal expansion was selected so the shock was located ahead of the hot-film. This lead to noise on the signal which we presume is due to shock oscillations in the divergent channel. Thus, one feature of the current design is the location of the shock behind the hot-films. 


\section{General Arrangement of the probe}

The previous considerations lead us to the following layout of the probe.

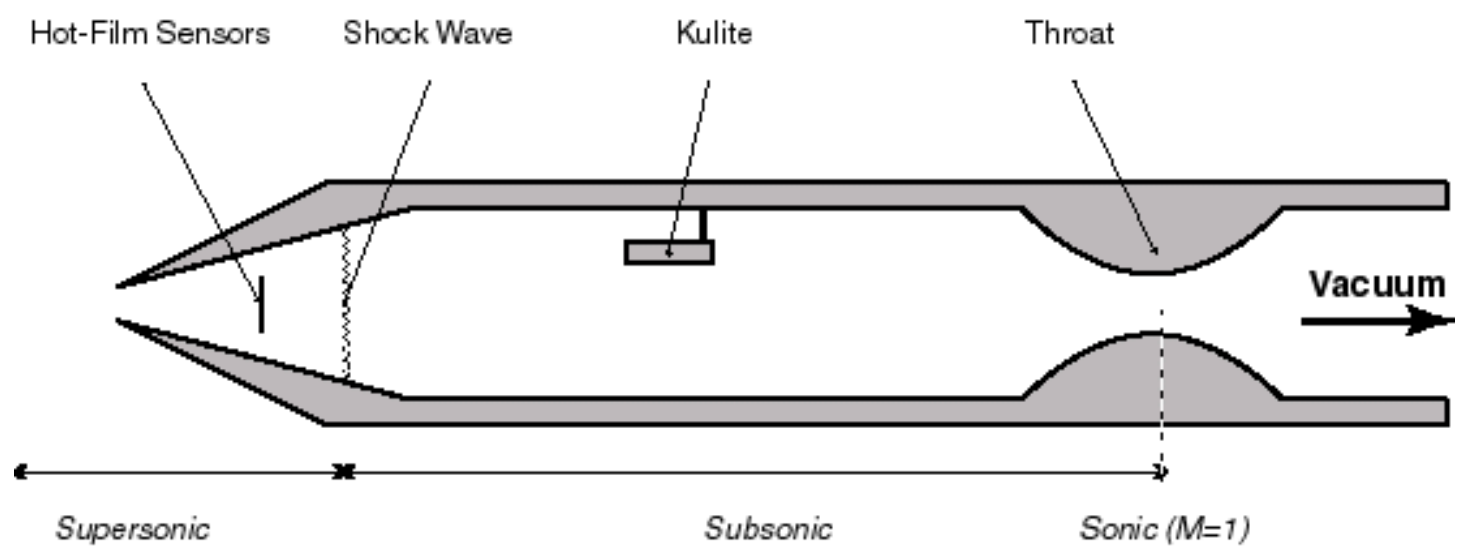

Figure 3 - Layout of the probe

The airflow entering the probe is accelerated in the divergent section and reaches a point where a shock wave occurs. Since we want the hot-films to operate in a supersonic flow, the sensor plane must be upstream of the shock whatever the inlet conditions are. The subsonic flow downstream of the shock is then accelerated through the throat by the vacuum downstream of the throat. If the backpressure is sufficiently low, the throat will be choked and sonic conditions will be reached at the throat. With these assumptions, we can use a one-dimensional analysis to evaluate the properties of the flow at any section of the probe.

\section{$1 D$ Analysis}

We are considering the flow inside the probe to be one dimensional and steady. This simple analysis allows us to determine what is the area required for the inlet orifice or for the throat orifice. It also gives an idea about the Mach number at the sensor plane and in 
front of the shock. This is important because when air and high Mach number are involved, condensation phenomena can occur ruining all of our measurements. In the analysis, we will consider four planes:

1. The inlet plane

2. The sensor plane

3. The shock plane

4. The throat plane

One can write the mass flow equation in its steady form for all the planes:

$$
(\rho U A)_{i n}=(\rho U A)_{h f}=(\rho U A)_{s h^{-}}=(\rho U A)_{s h^{+}}=(\rho U A)_{t h}
$$

(Eq. 8)

This can also be written as:

$$
\begin{aligned}
\frac{P_{t_{i n}}}{\sqrt{T_{t_{i n}}}} \sqrt{\frac{\gamma_{i n}}{R_{i n}}} A_{i n} M_{i n}\left(1+\frac{\gamma_{i n}-1}{2} M_{i n}^{2}\right)^{-\frac{1+\gamma_{i n}}{2\left(\gamma_{i n}-1\right)}} & =\frac{P_{t_{h f}}}{\sqrt{T_{t_{h f}}}} \sqrt{\frac{\gamma_{h f}}{R_{h f}}} A_{h f} M_{h f}\left(1+\frac{\gamma_{h f}-1}{2} M_{h f}^{2}\right)^{-\frac{1+\gamma_{h f}}{2\left(\gamma_{h f^{\prime}}-1\right)}} \\
& =\frac{P_{t_{s h^{-}}}}{\sqrt{T_{t_{s h^{-}}}}} \sqrt{\frac{\gamma_{s h^{-}}}{R_{s h^{-}}}} A_{s h} M_{s h^{-}}\left(1+\frac{\gamma_{s h^{-}}-1}{2} M_{s h^{-}}^{2}\right)^{-\frac{1+\gamma_{s h^{-}}}{2\left(\gamma_{s h^{-}}-1\right)}} \\
& =\frac{P_{t_{s h^{+}}}}{\sqrt{T_{t_{s h^{+}}}}} \sqrt{\frac{\gamma_{s h^{+}}}{R_{s h^{+}}}} A_{s h} M_{s h^{+}}\left(1+\frac{\gamma_{s h^{+}}-1}{2} M_{s h^{+}}^{2}\right)^{-\frac{1+\gamma_{s h^{+}}}{2\left(\gamma_{s h^{+}}-1\right)}} \\
& =\frac{P_{t_{h b}}}{\sqrt{T_{t_{h h}}}} \sqrt{\frac{\gamma}{R}} A_{t h}\left(\frac{\gamma+1}{2}\right)^{-\frac{1+\gamma}{2(\gamma-1)}}
\end{aligned}
$$

(Eq. 8a) 
With the assumptions that the flow is adiabatic ( $T_{t}$ is a constant) and that the gas composition is the same at every plane ( $\gamma$ and $R$ are constants), we can simplify the equations to Eq. 8 b:

$$
\begin{aligned}
P_{t_{i n}} A_{i n} M_{i n}\left(1+\frac{\gamma-1}{2} M_{i n}{ }^{2}\right)^{-\frac{1+\gamma}{2(\gamma-1)}} & =P_{t_{h f}} A_{h f} M_{h f}\left(1+\frac{\gamma-1}{2} M_{h f}{ }^{2}\right)^{-\frac{1+\gamma}{2(\gamma-1)}} \\
& =P_{t_{s h^{-}}} A_{s h} M_{s h^{-}}\left(1+\frac{\gamma-1}{2} M_{s h^{-}}{ }^{2}\right)^{-\frac{1+\gamma}{2(\gamma-1)}} \\
& =P_{t_{s h^{+}}} A_{s h} M_{s h^{+}}\left(1+\frac{\gamma-1}{2} M_{s h^{+}}{ }^{2}\right)^{-\frac{1+\gamma}{2(\gamma-1)}} \\
& =P_{t_{t h}} A_{t h}\left(\frac{\gamma+1}{2}\right)^{-\frac{1+\gamma}{2(\gamma-1)}}
\end{aligned}
$$

\section{(Eq. 8b)}

Once the internal geometry of the probe has been decided, i.e. the areas of the inlet orifice and of the throat are known, one can compute the Mach number at any location inside the probe. We are especially interested in the value of the Mach number at the sensor plane and the maximum Mach number reached inside the probe.

Using the final geometry of the probe $\left(A_{i n}=3.74610^{-6} \mathrm{~m}^{2}, A_{h f}=6.70110^{-6}\right.$ $\mathrm{m}^{2}, A_{t h}=7.40510^{-6} \mathrm{~m}^{2}$ ), we can evaluate the value of the Mach number at the sensor plane for different asssumed external flow Mach numbers. Since the sensor plane is located upstream of the shock, we have $P_{t_{i n}}=P_{t_{h f}}=P_{t_{s h^{-}}}$and the mass flow equation gives:

$$
A_{i n} M_{i n}\left(1+\frac{\gamma-1}{2} M_{i n}^{2}\right)^{-\frac{1+\gamma}{2(\gamma-1)}}=A_{h f} M_{h f}\left(1+\frac{\gamma-1}{2} M_{h f}{ }^{2}\right)^{-\frac{1+\gamma}{2(\gamma-1)}}
$$

(Eq. 9) 
Table 1 - Mach number at the sensor plane

\begin{tabular}{|c|c|c|}
\hline$M_{\text {in }}$ & Air & Helium \\
\hline 1 & 2.07 & 2.24 \\
\hline 2 & 2.64 & 2.87 \\
\hline 2.4 & 3.02 & 3.27 \\
\hline 3 & 3.62 & 3.92 \\
\hline
\end{tabular}

These values give an idea of what range of Mach number will be seen by the hot films. This is useful since we do not want to reach a very high Mach number ( $>4)$ at the sensor plane. This limitation makes one of the boundaries of the operating envelope of the probe.

The other operationnal boundaries come from the limitation of the maximum Mach number inside the probe. We want that at any point inside the probe the Mach number is lower than 4.5. This limitation is due to the condensation phenomena that happen when air reaches high Mach Number. The highest Mach number is found in front of the shock. We thus have to compute the strength and position of the shock. This can be done using the ratio $P_{t_{s h^{+}}} / P_{t_{s h^{-}}}$.

To find this ratio, we write the mass flow equation:

$$
P_{t_{i n}} A_{i n} M_{i n}\left(1+\frac{\gamma-1}{2} M_{i n}{ }^{2}\right)^{-\frac{1+\gamma}{2(\gamma-1)}}=P_{t_{t h}} A_{t h}\left(\frac{\gamma+1}{2}\right)^{-\frac{1+\gamma}{2(\gamma-1)}}
$$

(Eq. 10)

which leads to: 


$$
\frac{P_{t_{s h^{+}}}}{P_{t_{s h^{-}}}}=\frac{A_{i n}}{A_{t h}} M_{i n}\left(\frac{2+(\gamma-1) M_{i n}{ }^{2}}{\gamma+1}\right)^{-\frac{1+\gamma}{2(\gamma-1)}}
$$

(Eq. 10a)

This ratio is also linked to the Mach number upstream of the shock $M_{s h^{-}}$by the relation:

$$
\frac{P_{t_{s h^{+}}}}{P_{t_{s h^{-}}}}=\frac{\left[\frac{(\gamma+1) M_{s h^{-}}{ }^{2}}{2+(\gamma-1) M_{s h^{-}}{ }^{2}}\right]^{\frac{\gamma}{\gamma-1}}}{\left[\frac{2 \gamma}{\gamma+1} M_{s h^{-}}{ }^{2}-\frac{\gamma-1}{\gamma+1}\right]^{\frac{1}{\gamma-1}}}
$$

(Eq. 11)

Table 2 - Pressure ratio across the shock and Mach number upstream of the shock

\begin{tabular}{|c|c|c|c|}
\hline \multicolumn{2}{|c|}{$M_{\text {in }}$} & Air & Helium \\
\hline \multirow{3}{*}{1} & $P_{s_{s h^{+}}} / P_{t_{s h^{-}}}$ & 0.559 & 0.559 \\
\cline { 2 - 4 } & $M_{s h^{-}}$ & 2.355 & 2.557 \\
\hline \multirow{2}{*}{2} & $P_{t_{s h^{+}}} / P_{t_{s h^{-}}}$ & 0.331 & 0.366 \\
\cline { 2 - 4 } & $M_{s h^{-}}$ & 2.989 & 3.264 \\
\hline \multirow{3}{*}{2.4} & $P_{s_{s h^{+}}} / P_{t_{s h^{-}}}$ & 0.233 & 0.28 \\
\cline { 2 - 4 } & $M_{s h^{-}}$ & 3.398 & 3.715 \\
\hline \multirow{3}{*}{3} & $P_{s_{s h^{+}}} / P_{t_{s h^{-}}}$ & 0.132 & 0.187 \\
\cline { 2 - 4 } & $M_{s h^{-}}$ & 4.059 & 4.445 \\
\hline
\end{tabular}

We also have to verify that the hot films are in a supersonic flow as supposed and determine the distance between the sensor plane and the plane where the shock occurs. Evaluate the area where the shock wave forms. Using the mass flow equation again, we find Eq. 12: 


$$
A_{s h}=A_{i n} \frac{M_{i n}}{M_{s h^{-}}}\left(\frac{1+\frac{\gamma-1}{2} M_{i n}{ }^{2}}{1+\frac{\gamma-1}{2} M_{s h^{-}}{ }^{2}}\right)^{-\frac{1+\gamma}{2(\gamma-1)}}
$$

(Eq. 12)

The internal geometry of the divergent is known. Consequently, it is easy to find the distance between the shock and the hot-films $d=\frac{\sqrt{A_{s h}}-\sqrt{A_{h f}}}{\sqrt{\pi} \tan \left(10^{\circ}\right)}$

Table 3 - Position of the shock and distance to the sensor plane

\begin{tabular}{|c|c|c|c|}
\hline \multicolumn{2}{|c|}{$M_{\text {in }}$} & Air & Helium \\
\hline \multirow{2}{*}{1} & $A_{s h}$ (in. $^{2}$ ) & $1.340 \mathrm{e}-2$ & $1.289 \mathrm{e}-2$ \\
\cline { 2 - 4 } & $d$ (in.) & $4.42 \mathrm{e}-2$ & $3.72 \mathrm{e}-2$ \\
\hline \multirow{2}{*}{2} & $A_{s h}$ (in. $\left.^{2}\right)$ & $1.443 \mathrm{e}-2$ & $1.351 \mathrm{e}-2$ \\
\cline { 2 - 4 } & $d$ (in.) $^{2}$ & $5.82 \mathrm{e}-2$ & $4.57 \mathrm{e}-2$ \\
\hline \multirow{2}{*}{2.4} & $A_{s h}$ (in. $^{2}$ ) & $1.492 \mathrm{e}-2$ & $1.376 \mathrm{e}-2$ \\
\cline { 2 - 4 } & $d$ (in.) $^{2}$ & $6.47 \mathrm{e}-2$ & $4.92 \mathrm{e}-2$ \\
\hline \multirow{2}{*}{3} & $A_{s h}$ (in. $\left.{ }^{2}\right)$ & $4.55 \mathrm{e}-2$ & $1.405 \mathrm{e}-2$ \\
\cline { 2 - 4 } & $d$ (in.) & $7.22 \mathrm{e}-2$ & $5.32 \mathrm{e}-2$ \\
\hline
\end{tabular}


Finally, we can plot the operating envelope of the chosen probe design.

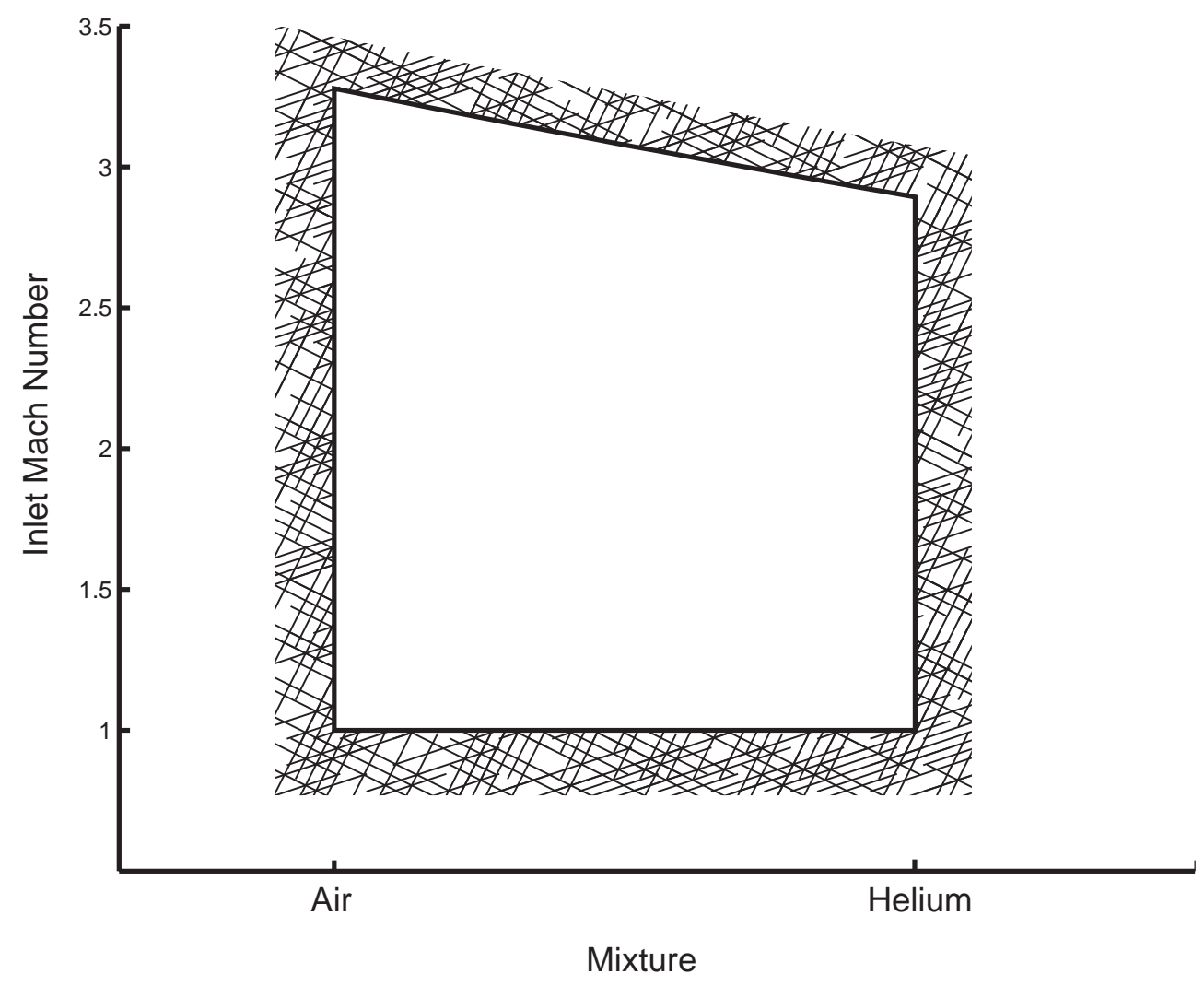

Figure 4 - Operating envelope of the probe

Of course, one can change the area ratios and recompute the operating envelope. The design selected was the result of numerous exercises of this type. Other items that influenced the final design were the size of the hot-film and pressure sensors and the sampling volume of the probe.

\section{Estimation of the natural frequency of the probe}

The frequency response of a probe depends not only on the frequency response of the transducers used but also on the design of the probe itself. The frequency response of the hot-films and the pressure transducer selected approximate $100 \mathrm{kHz}$. Consequently it is important to evaluate the time lag due to the internal volume of the probe. A simple 
analysis from Konkin, Lebiga and Zinoviev (1996) gives an easy way to evaluate this time lag. It was found that, for Air - Helium mixture the worst case was for a 100\% air mixture. The frequency of the probe is given by Eq. 13:

$$
a=\frac{\beta R \sqrt{T_{t}}}{V} q\left(M_{t h}\right) A_{t h}
$$

(Eq. 13)

where:

$$
\beta=\sqrt{\frac{\gamma}{R}\left(\frac{2}{\gamma+1}\right)^{\frac{\gamma+1}{\gamma-1}}}
$$

\section{(Eq. 14)}

$$
q(M)=\left(\frac{\gamma+1}{2}\right)^{\frac{\gamma+1}{2(\gamma-1)}} \frac{M}{\sqrt{1+\frac{\gamma-1}{2} M^{2}}}\left(1+\frac{\gamma-1}{2} \frac{M^{2}}{1+\frac{\gamma-1}{2} M^{2}}\right)^{\frac{1}{\gamma-1}}
$$

(Eq. 15)

Using $T_{t}=300 \mathrm{~K}$ and the final geometry of the probe, we find that $V=9.31 \mathrm{e}-8 \mathrm{~m}^{3}$ and $a=37 \mathrm{kHz}$. Consequently, the internal volume shouldn't be a limitation on the desired frequency response of the probe of $1 \mathrm{kHz}$.

\section{Construction}

The physical design of the probe was not an easy task. The 1D analysis gave some dimensions for the throat and the inlet orifice, the location of the sensor plane, etc. We also had to cope with the size of the different parts (Kulite, Hot film probe) and the ease of machining. This required a lot of interaction with the machine shop and the companies 
that designed hot-films probe to reach a viable design. Some parts were extremely small, and complex machining was out of question. Thus, the design had to be kept as simple as possible.

We also had to keep in mind that the hot-films could break and that we should have to repair them. This repair issue was taken into account by designing a removable cap. This cap serves as the inlet and divergent section of the probe. Its external angle is $30^{\circ}$ which is enough to keep the shock attached when the free stream Mach number is 3.The internal angle is only $10^{\circ}$. A low value is used to reduce separation phenomena near the shock inside the divergent channel and also to increase the distance between the shock and the sensor plane.
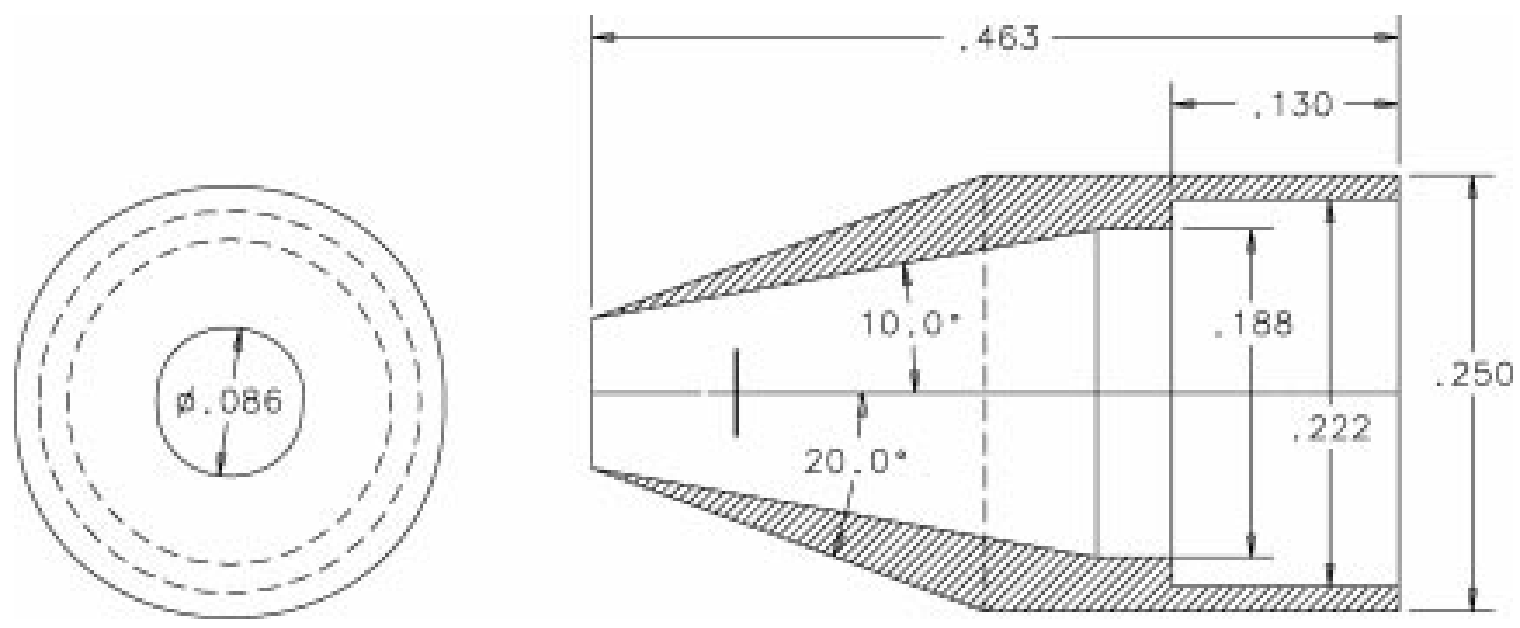

Figure 5 - Removable cap design

The throat was a little more complex to design. The part that creates the contraction should not be too far downstream. If placed too far downstream, the internal volume of the probe will increase, and the natural frequency of the probe decrease. The other problem we faced was that the Kulite and the hot film probe had to be held firmly in place. The solution was to machine a part that will have two functions:

1. Reduce the cross flow section of the probe (serve as a throat)

2. Hold the Kulite and the hot-film probe Two designs were tested and are shown in Figure 6. 

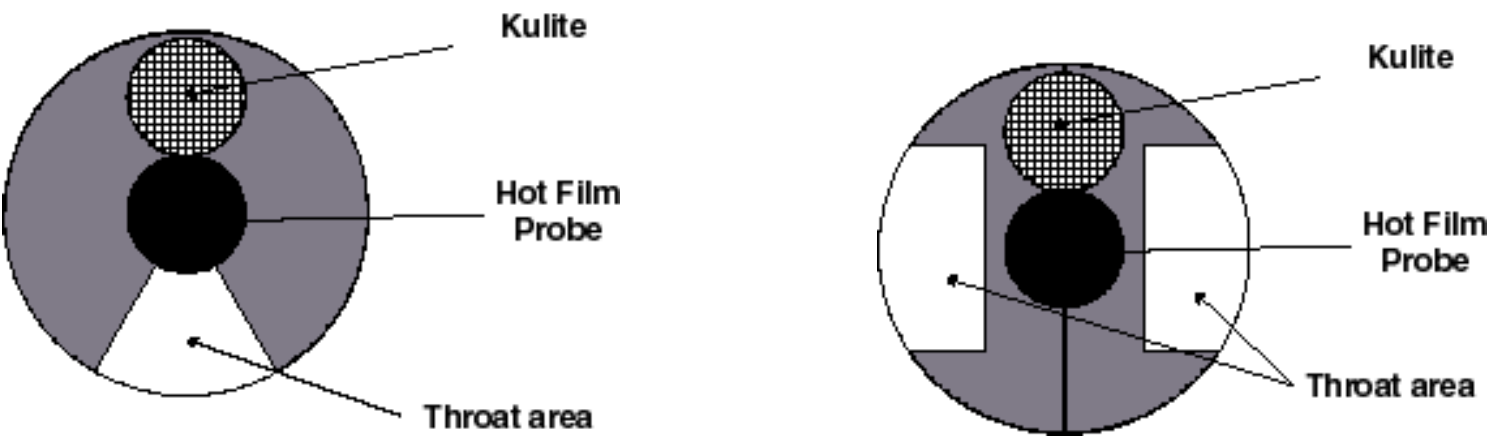

Figure 6 - Possible geometries of the throat insert

The second one appeared to work better, maybe because of its more symmetric design.
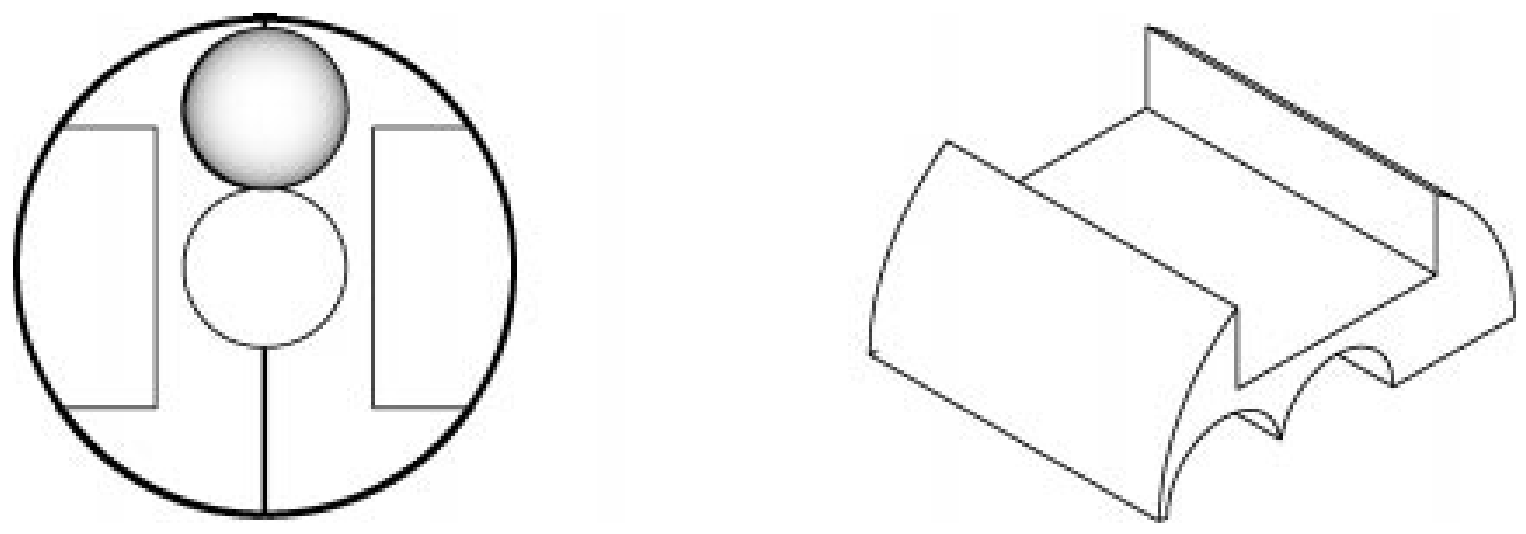

Figure 7 - Final geometry of the throat insert

Finally, the electrical wires of the Kulite were pulled out of the probe down the vacuum tube.

The final design of the probe is shown in Figure 8. The external diameter of the probe is only 0.25 in. 


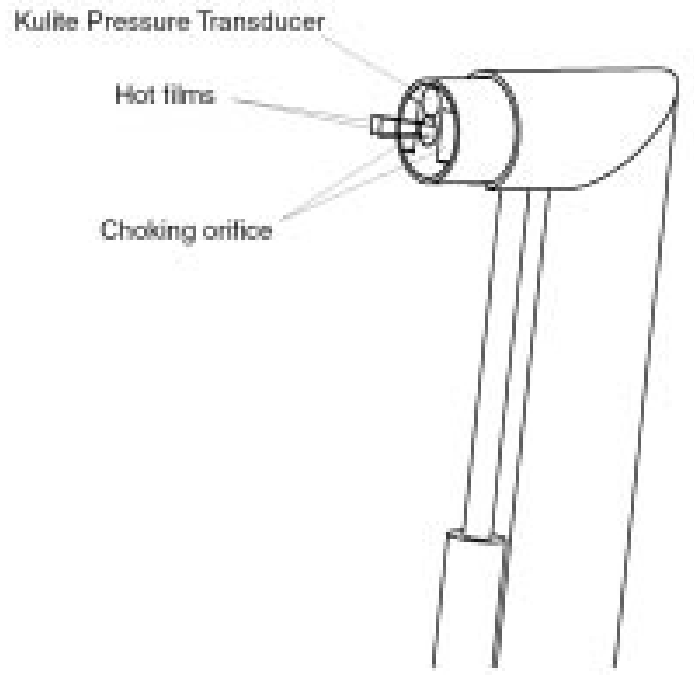

View of the probe without the cap

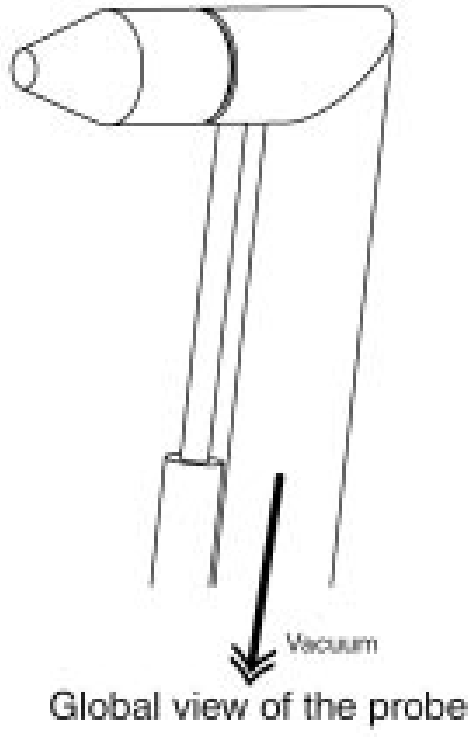

Global view of the probe

Figure 8 - Final design of the probe 


\section{Data Acquisition and Calibration}

We have seen previously that two constants, $a$ and $m$ (see Eq. 5a), were needed to determine the concentration. These two constants depend on the concentration only. Consequently, we must first go through a calibration phase before beginning any other measurements especially wind-tunnel tests.

\section{The data acquisition system}

The same data acquisition system was used for both the calibration and the wind tunnel measurements. It is capable of very high sampling rate (up to $1 \mathrm{MHz}$ ) even though we will only use rates up to $10 \mathrm{kHz}$.

The figure below shows the data acquisition system used to record data from the probe.

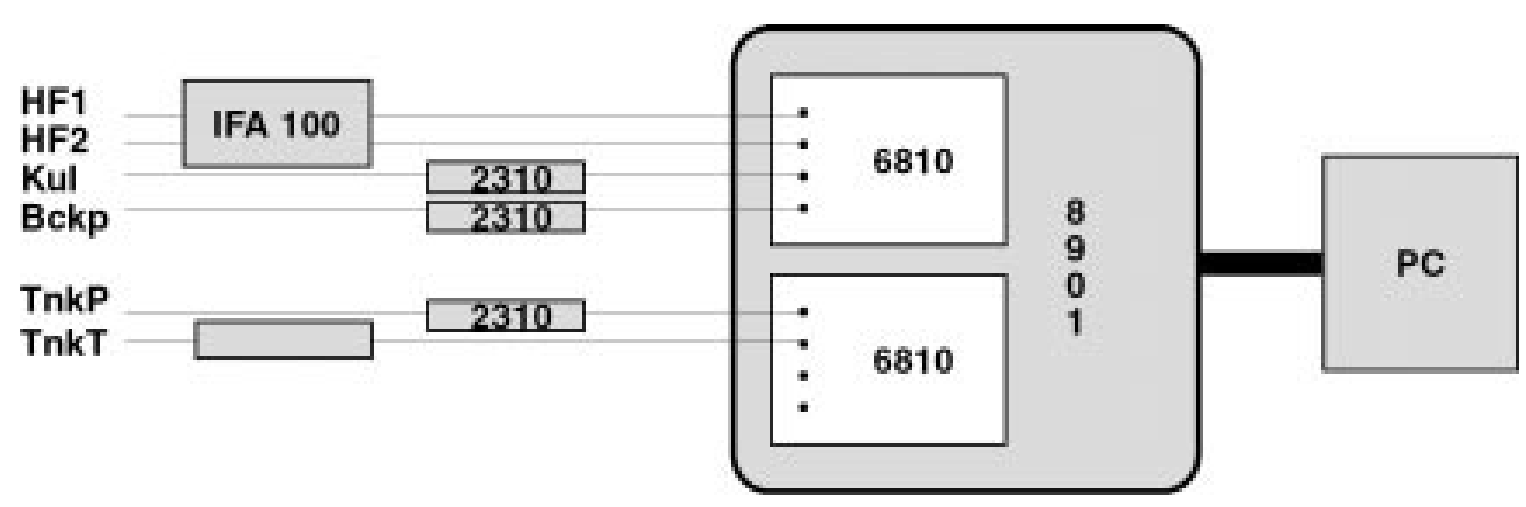

Figure 9 - Data acquisition system

It is based on two Lecroy 6810 digitizers. The signals that comes from the TSI IFA 100, from the Micromeasurements 2310 amplifiers and the thermocouple box are digitized and then recorded in the memory of the Lecroy. The samples are finally transferred to the PC via a GPIB interface and are stored on disk.

In the box that forms the Lecroy, we can find two components: the 8901 GPIB interface and the 6810 digitizers. The 8901 interface allows the communications between the PC 
and the digitizers. The PC is running Catalyst. This software allows the configuration of the 6810 , i.e. sampling rate, number of samples to acquire, voltage range, trigger options, etc... It also displays the traces of the acquired signals on the screen. Each 6810 digitizer can acquire up to 4 signals simultaneously at sampling rates up to $5 \mathrm{MHz}$ ( $1 \mathrm{MHz}$ if the 4 channels are used). The acquired data are digitized with a 12-bit resolution. Once the digitizer is armed, the data are acquired continuously and stored in the 6810 memory. When the memory is full, the new data overwrites the old data. When a trigger signal is received, this overwrite process is disabled. This technique allows one to record data before the trigger signal actually happens, for example before a moving shock reaches a transducer.

The TSI IFA (Intelligent Flow Analyzer) is a device designed for thermal anemometry. It contains 2 constant temperature anemometer and a built-in signal conditioner (offset, gain and high-pass filter). It can work with hot-wires and hot-films. It also has a built-in square wave generator to tune the frequency response of the sensors.

\section{Calibration}

The calibration phase is necessary to find the experimental constants, $a$ and $m$, used for the hot films measurements. Unfortunately, these constants depend on the concentration of the mixture. Consequently, we need to determine these constants for several values of the concentration. For that purpose, measurements must be made with a known mixture, and the constants will be deduced from them. The calibration tank was built to do these measurements

The easiest way to make measurements with a known mixture is to let the probe aspirate the gas from a tank where the mixture has been previously prepared. Our tank uses a similar principle as shown in Figure 10. 


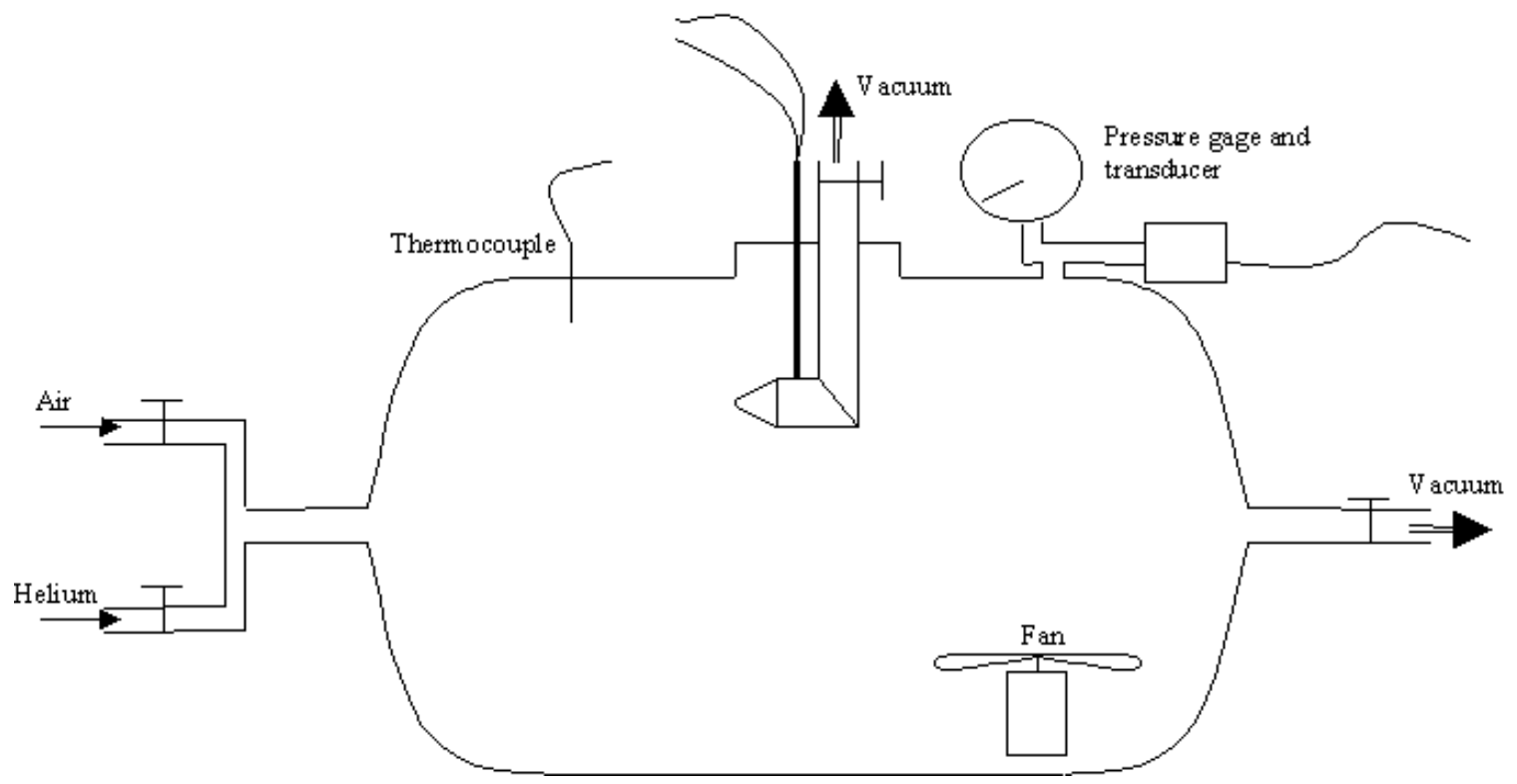

Figure 10 - Calibration tank layout

The tank is connected to an air cylinder and a helium cylinder. Valves allow filling the tank with the desired quantities of each gas. A vacuum pump is also connected to the tank. It is used to empty the tank of any residual gases before starting a new mixture. This precaution is necessary because remains of a previous mixture could lead to major errors in the value of the concentration. The tank is also equipped with a pressure gage that permits monitoring the quantities of gas introduced into the tank. A pressure transducer and a thermocouple record the pressure and temperature changes when the probe aspirates the mixture from the tank. A tiny fan was also introduced into the tank to enhance the mixing between the two gases, as we found, the air and helium had the tendency to form layers for the higher concentrations of helium. The probe sits inside the tank and is consequently surrounded by a mixture at a known concentration. Particular care was taken to make sure that there were no leaks in this setup. As a consequence, the mixture that is aspirated by the probe is the one prepared in the tank.

In order to get the values of the constants for a given concentration, we first need to have some measurements for this particular concentration. The following procedure explains the different steps to get a set of data for a given concentration:

1. Make sure that all the valves are closed. 
2. Open the valve to the vacuum pump. When a vacuum is established in the tank, close the valve.

3. Slowly fill the tank with the necessary quantities of air and He to achieve the desired concentration. The concentration can be easily found; it is the ratio $\frac{P_{H e}}{P_{H e}+P_{A i r}}$. For example, to create a $60 \%$ mixture, we can fill the tank with 30 psi of helium and then complete with air until we reach 50 psi.

4. Make sure that the data acquisition system is ready (Hot-films are running, Lecroy is armed, etc...)

5. Completely open the valve that connects the probe to the vacuum tank and manually trigger the Lecroy. The Lecroy starts digitizing and storing the data.

6. When the Lecroy is finished sampling, close the valve.

7. Wait a while until the pressure in the vacuum tank is low enough. Then, go back to step 4 in order to do some more measurements for the same concentration, but a lower tank pressure. It is recommended to do that 5 or 6 times.

The result of this procedure should give traces that look like those in the following figures (Figure 11):

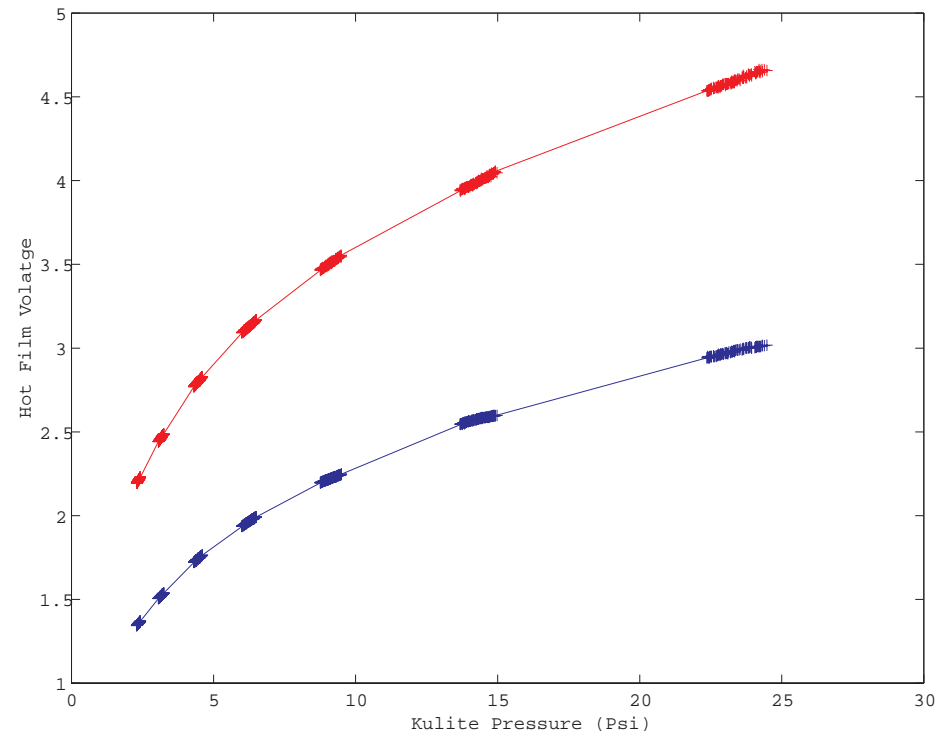

Figure 11 - Typical calibration curves for a given concentration 
We ran this procedure for several mixtures $(0 \%, 20 \%, 40 \%, 60 \%, 80 \%$ and $100 \%)$ and obtained the following curves (Figure 12,Figure 13).

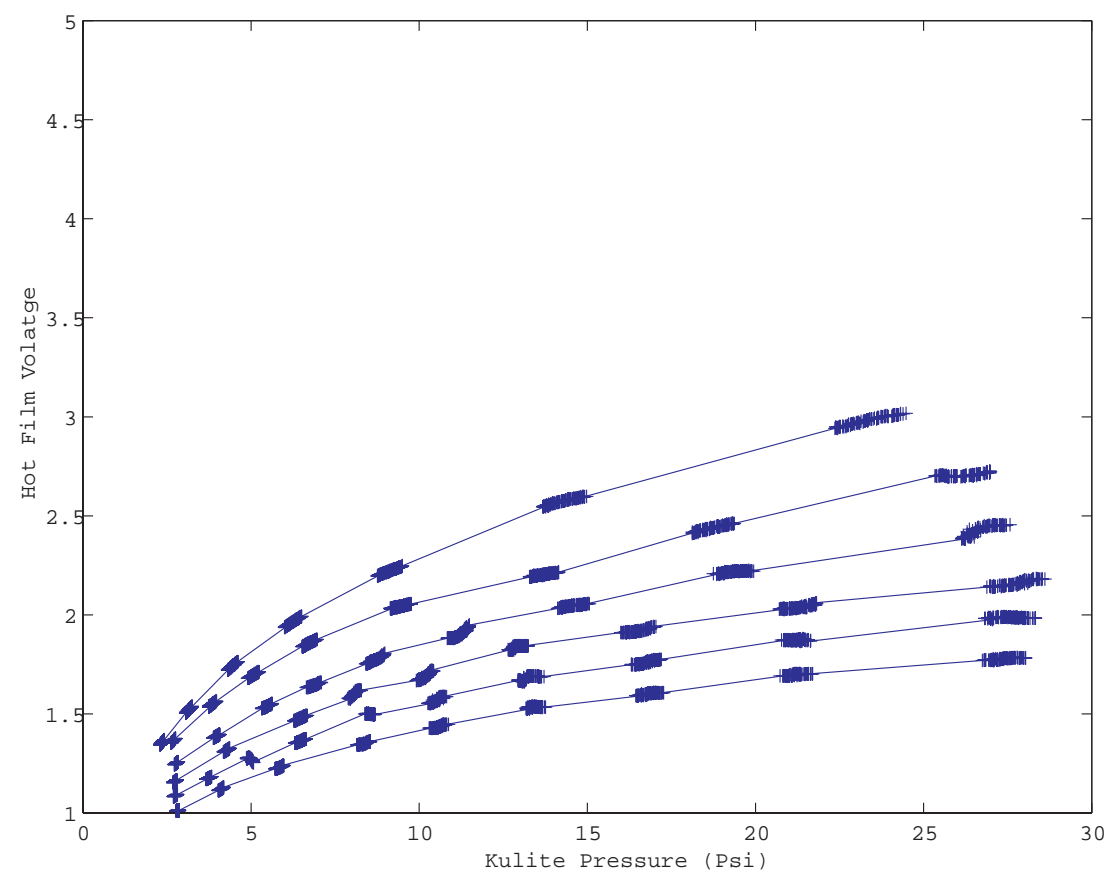

Figure 12 - Calibration curves (low overheat hot-film)

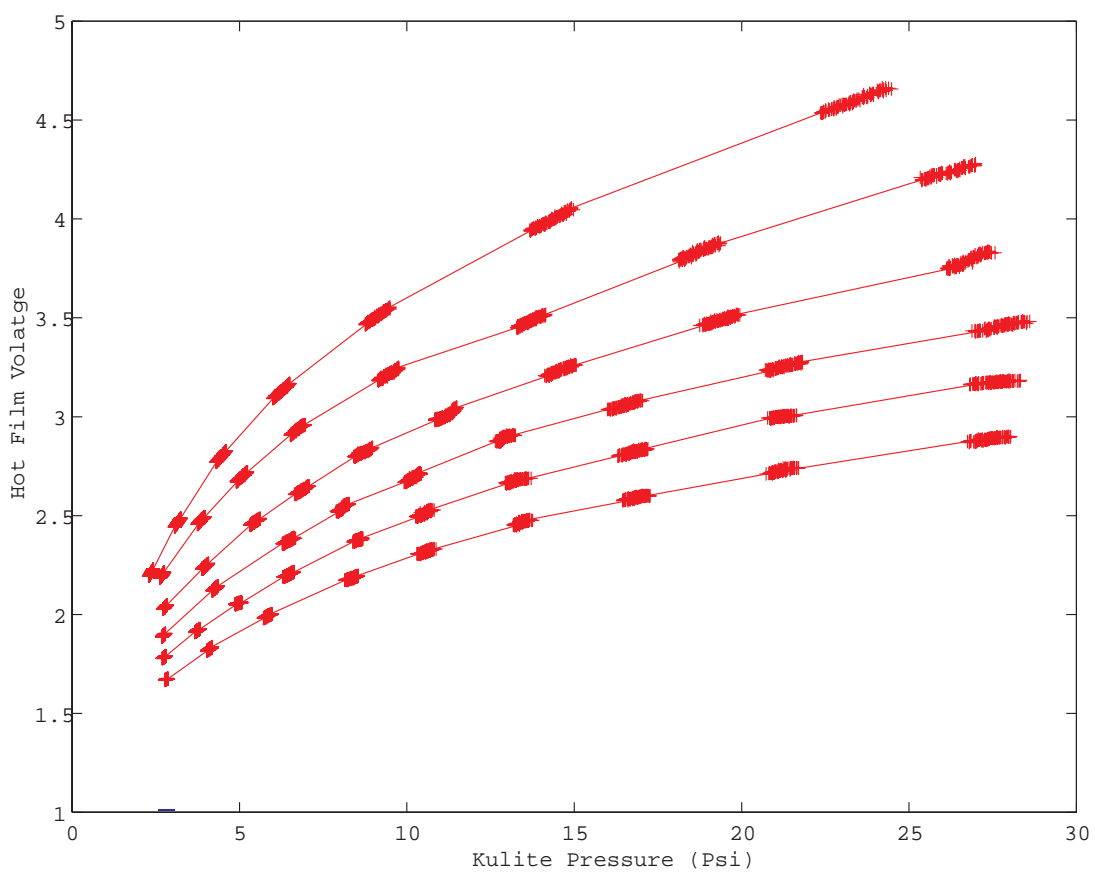

Figure 13 - calibration curves (high overheat hot-film) 


\section{Determination of the calibration constants}

The principle of operation made clear that the voltage of the anemometers follows

$$
V_{i}^{2}=\mathrm{A}_{i} N u\left(T_{w_{i}}-T_{e}\right)
$$

(Eq. 16)

Since the physical properties of the mixture (viscosity, thermal conductivity...) hidden in $A_{i}$ and $N u$ are evaluated at the stagnation temperature $T_{t}$, this relation involves two unknown temperatures: $T_{e}$ and $T_{t}$. However, the recovery factor $\eta$ defined in Smits, Hayakawa and Muck (1983) as

$$
\eta=\frac{T_{e}}{T_{t}}
$$

(Eq. 17)

can be used to evaluate $T_{t}$ and the physical properties from the recovery temperature $T_{e}$. As a consequence, the only unknown is $T_{e}$ which is determined from the voltages of the anemometers:

$$
T_{e}=\frac{\mathrm{A}_{2} V_{1}^{2} T_{w_{2}}-\mathrm{A}_{1} V_{2}^{2} T_{w_{1}}}{\mathrm{~A}_{2} V_{1}^{2}-\mathrm{A}_{1} V_{2}^{2}}
$$

(Eq. 18)

In order to find the appropriate value of $\eta$, we used the experimental data. In our calibration setup, the thermocouple records the temperature inside the tank, which is also the stagnation temperature $T_{t}$. The recovery temperature $T_{e}$ comes from the anemometers. The stagnation temperature was found to be nearly constant on the 52 sets 
of data we recorded. It only varied between $293 \mathrm{~K}$ and $296 \mathrm{~K}$. On the other hand, $T_{e}$ was found to vary in a wider range, from $255 \mathrm{~K}$ to $290 \mathrm{~K}$. The value of $\eta$ was found by computing the ratio $\frac{T_{e}}{T_{t}}$ for every sample of every concentration and averaging them all together. The resulting value of the recovery factor is

$$
\eta=0.94
$$

and is consistent with the values found in the literature (Smits, Hayakawa and Muck (1983)).

The operation of the probe is based on the relation that exists between the Reynolds number and the Nusselt number. As we seen before, we have chosen the relation

$$
N u=a \operatorname{Re}^{m}
$$

\section{(Eq. 19)}

where $a$ and $m$ are experimentally determined constants that depend on the concentration only. These constants are found by curve fitting the data from a given concentration with the previous law. If we take the logarithm of this relation, we get:

$$
\ln (N u)=\ln \left(a \operatorname{Re}^{m}\right)=m \ln (\operatorname{Re})+\ln a
$$

(Eq. 20)

A least-squares curve fitting approach looks appropriate to find $m$ and $\ln a$. Some calculus lead us to the following relations (Eq. 21 and Eq. 22)(where $N$ is the number of data points): 


$$
m=\frac{\sum \ln \operatorname{Re} \ln N u-\frac{1}{N} \sum \ln \operatorname{Re} \sum \ln N u}{\sum(\ln \mathrm{Re})^{2}-\frac{1}{N}\left(\sum \ln \mathrm{Re}\right)^{2}}
$$

(Eq. 21)

$$
\begin{gathered}
\ln a=\frac{\sum \ln N u}{N}-\frac{\sum \ln \mathrm{Re}}{N} b \\
a=\exp (\ln a)=\exp \left(\frac{\sum \ln N u}{N}-\frac{\sum \ln \operatorname{Re}}{N} b\right)
\end{gathered}
$$

(Eq. 22)

In order to apply this method, we need to compute $N u$ and Re for every sample. The value of the Nusselt number comes from the voltage of the anemometers:

$$
N u=\frac{\mathrm{A}_{2} V_{1}^{2}-\mathrm{A}_{1} V_{2}^{2}}{\mathrm{~A}_{1} \mathrm{~A}_{2}\left(T_{w_{1}}-T_{w_{2}}\right)}
$$

(Eq. 23)

The Reynolds number must be calculated at the stagnation conditions. However, these conditions are also the total conditions at the sensor plane. Consequently, we have to evaluate:

$$
\operatorname{Re}=\frac{\rho_{h f} U_{h f} d}{\mu}
$$

\section{(Eq. 24)}

Unfortunately, we do not know $\rho_{h f} U_{h f}$. However, we know that the mass flow at the sensor plane is equal to the mass flow at the throat. 


$$
\rho_{h f} U_{h f} A_{h f}=\rho_{t h} U_{t h} A_{t h}
$$

(Eq. 25)

Since the throat is choked, the mass flow has a known value.

$$
\rho_{t h} U_{t h} A_{t h}=\frac{P_{t_{t h}}}{\sqrt{T_{t_{t h}}}} \sqrt{\frac{\gamma}{R}} A_{t h}\left(\frac{1+\gamma}{2}\right)^{-\frac{\gamma+1}{2(\gamma-1)}}
$$

(Eq. 26)

With the additional assumption that the flow is adiabatic $\left(T_{t_{t h}}=T_{t}\right)$ and that the total pressure at the throat is the pressure measured by the Kulite, we finally obtain:

$$
\operatorname{Re}=\frac{A_{t h}}{A_{h f}} \frac{d}{\mu} \frac{P_{k u l}}{\sqrt{T_{t}}} \sqrt{\frac{\gamma}{R}}\left(\frac{1+\gamma}{2}\right)^{-\frac{\gamma+1}{2(\gamma-1)}}
$$

(Eq. 27)

We applied this procedure for each concentration level we had. The resulting constants are:

Table 4 - Calibration Constants

\begin{tabular}{|c|c|c|}
\hline $\mathrm{X}$ & $a$ & $m$ \\
\hline 0 & 4.19743 & 0.469548 \\
\hline 0.2 & 2.80557 & 0.488318 \\
\hline 0.4 & 2.24425 & 0.503715 \\
\hline 0.6 & 2.04011 & 0.518208 \\
\hline 0.8 & 2.10811 & 0.537137 \\
\hline 1.0 & 2.42563 & 0.592056 \\
\hline
\end{tabular}

A $\mathrm{C}++$ code is presented in Appendix B. 


\section{Experiments}

\section{Facilities}

After calibrating the probe, we needed to make experiments to determine if the probe works correctly, especially if the probe is working in supersonic flows. We also want to check if the composition we find by reducing the data corresponds to the real composition of the mixture. Finally, we want to determine the frequency response of the probe. We had two possible ways to test the probe in a supersonic flow: either the supersonic wind tunnel or the hypersonic wind tunnel, both at Virginia Tech. The supersonic tunnel had the advantage of having a long runtime (about 10 seconds) whereas the hypersonic wind tunnel could only run for a few milliseconds. However, the hypersonic wind tunnel presents a lot of advantages.In particular, it has the possibility to be run with different gases or mixtures of gases at a wide range of total pressure.

The Virginia Tech hypersonic wind tunnel was originally designed and built in Russia by Dr. V. Zvegintsev of the Institute of Theoretical and Applied Mechanics of the Russian Academy of Sciences in Novosibirsk.; see Figure 14

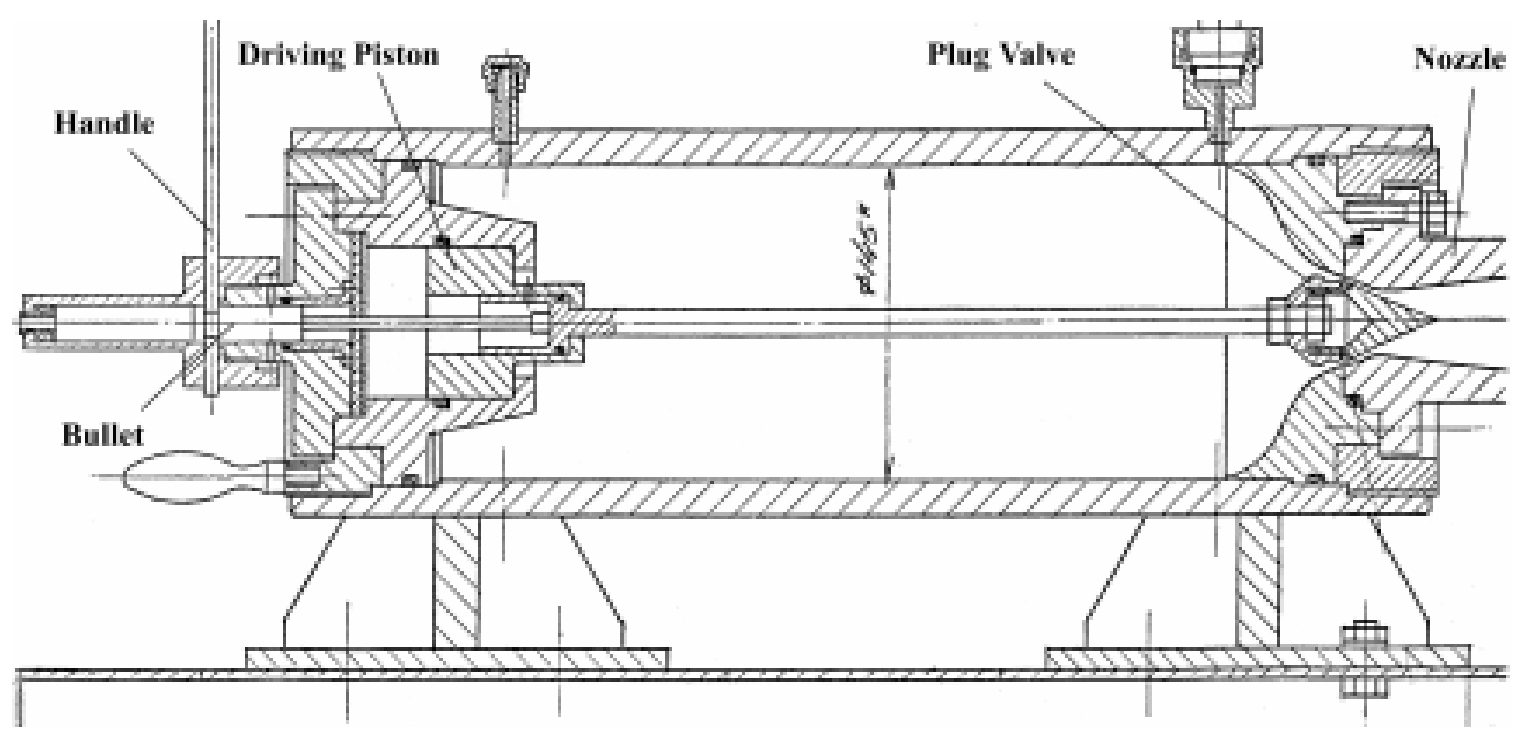

Figure 14 - Hypersonic Wind Tunnel Layout 
The major advantage of this facility is its simplicity. The pressure source for the gas is bottled air or other gases, and, since the tunnel exhausts at atmospheric pressure, no complicated setup is required. Running the tunnel is also very simple. It basically consists in lifting the handle. The driving piston can now move backward with the help of the pressure forces and pull the plug valve out of the entrance of the nozzle. The gas can then rush through the nozzle and into the test section. The process of opening this fast-acting valve only takes about 4 to 5 milliseconds.

The other advantage is its flexibility. It can be easily configured to run at Mach numbers from 2 to 7. The initial plenum pressure can also vary in a wide range, from $0.2 \mathrm{MPa}$ to $20 \mathrm{MPa}$. A heater can be installed to increase the temperature of the gas inside the plenum chamber to $850{ }^{\circ} \mathrm{K}$. This is very useful for higher Mach numbers (4 - 7), where it prevents condensation phenomena. The run-time of this facility depends on the nozzle used and the pressure of the plenum chamber. Under some conditions it can reach 130 milliseconds.

\section{Test Setup}

The hypersonic wind tunnel was configured to run with the Mach 2 nozzle for these tests. A special support was needed to hold the probe in the test section. It also helps to reduce the vibrations and the mechanical constraints on the probe. The inlet of the probe is located in the center of the jet and at about $5 \mathrm{~cm}$ downstream the exit of the nozzle. Due to the Kulite transducer range, which was rated for $50 \mathrm{psi}$, we were not able to run the tunnel with the high pressure and were limited to about 80 psi. Also, at Mach 2, the dynamic pressure and thus loads are large.

\section{Test procedure}

The procedure to acquire one set of data can be described by the following steps:

1. Push the bullet and the plug to close the settling chamber. Make sure the handle is in the groove of the bullet. 
2. Establish a vacuum inside the settling chamber.

3. Fill the settling chamber with the desired mixture and a pressure of 65 Psi. The tunnel is now ready to run.

4. Make sure that the data acquisition system is ready. The Lecroy should be configured for automatic triggering. The condition is that the signal from the high overheat anemometer goes over $2.58 \mathrm{~V}$

5. Arm the Lecroy.

6. Open the valve from the probe to the vacuum tank. The probe will begin to suck air from the test section. The Lecroy should not trigger.

7. Release the handle. The tunnel starts. As soon as the Lecroy finishes acquiring data, close the valve.

8. Reduce the data to have the trace of the mole concentration as a function of time.

\section{Data reduction}

The process of reducing the probe data uses a direct technique. A computer program implements a dichotomy to find the concentration. For each sample, we have the two voltages from the anemometers and the pressure from the Kulite transducer. Starting with the interval $[\mathrm{Xl} ; \mathrm{Xh}]$ (initially $\mathrm{Xl}=0.0$ and $\mathrm{Xh}=1.0$ ), we can set the concentration to $\mathrm{Xm}$, the average value of $\mathrm{Xl}$ and $\mathrm{Xh}$. We then use the data from the probe to compute the following quantities:

1. The recovery temperature from the hot-film voltages

2. The Nusselt number from the hot-film voltages

3. The Reynolds number from the Kulite transducer

This is basically the same as the calibration phase. We also compute $a \operatorname{Re}^{m}$, which is another expression of $N u$. If the constants $a$ and $m$ do not exist for the concentration $\mathrm{Xm}$, we linearly interpolate their value from the nearest available concentration levels. We then compare $N u$ and $a \operatorname{Re}^{m}$. If $N u$ is greater than $a \operatorname{Re}^{m}$, the concentration is greater than Xm and we repeat the process with the interval $[\mathrm{Xm}, \mathrm{Xh}]$. On the other hand, if $N u$ is lower than $a \operatorname{Re}^{m}$, the concentration is lower and the new interval is $[\mathrm{Xl}, \mathrm{Xm}]$. 
We repeat this procedure until the desired precision. We actually took Xh-Xl<=1\%.

A C++ code for data reductioon is presented in Appendix B.

\section{Results}

After the calibration phase, we wanted to test the probe in a more realistic way. That means in a supersonic flow. In order to verify that the probe was working properly, we want to compare the composition given by the data of the probe with the real composition of the mixture. For that purpose, we ran tests with a helium concentration of $0 \%, 25 \%$, $50 \%$ and $100 \%$ in the hypersonic tunnel. For each test, we also recorded some milliseconds of data prior to the triggering of the Lecroy. As a consequence, we should see some changes of the concentration with time. We even expect to see, during the few milliseconds the run lasts, a step change.

The following plots shows the signals from the probe (Kulite pressure and anemometer voltages) and the resulting concentration trace for the concentrations $0 \%, 25 \%, 50 \%$ and $100 \%$.

The resulting concentration measurements look very noisy. We first thought that it was the consequence of a phase shift between the hot-film signals and the Kulite pressure signal. Consequently, in order to decrease these fluctuations, we tried to introduce a time lag between the signals to account for the physical separation of the transducers. The results were not what was expected; the signal became even noisier. 

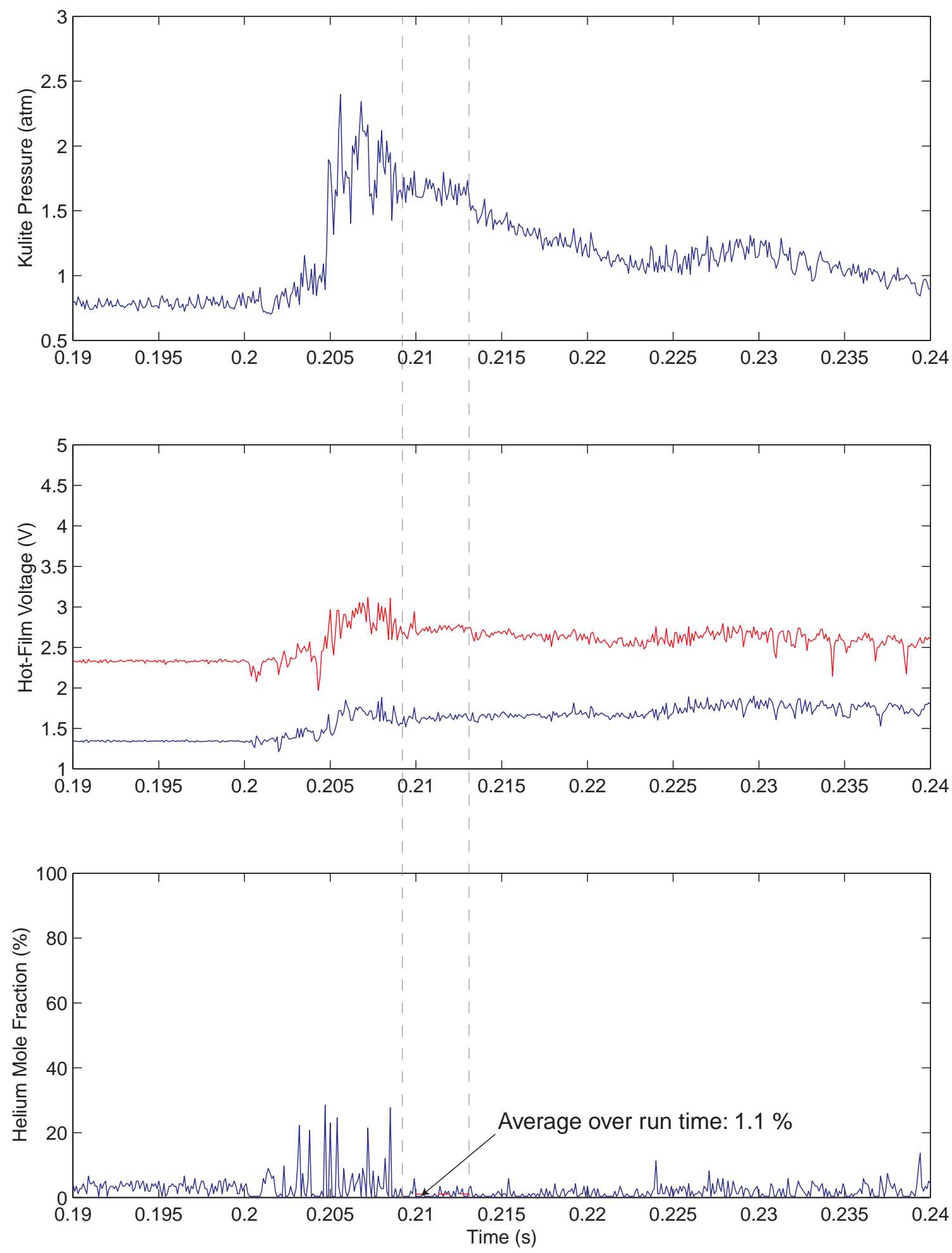

Figure 15 - Concentration: 0\% 

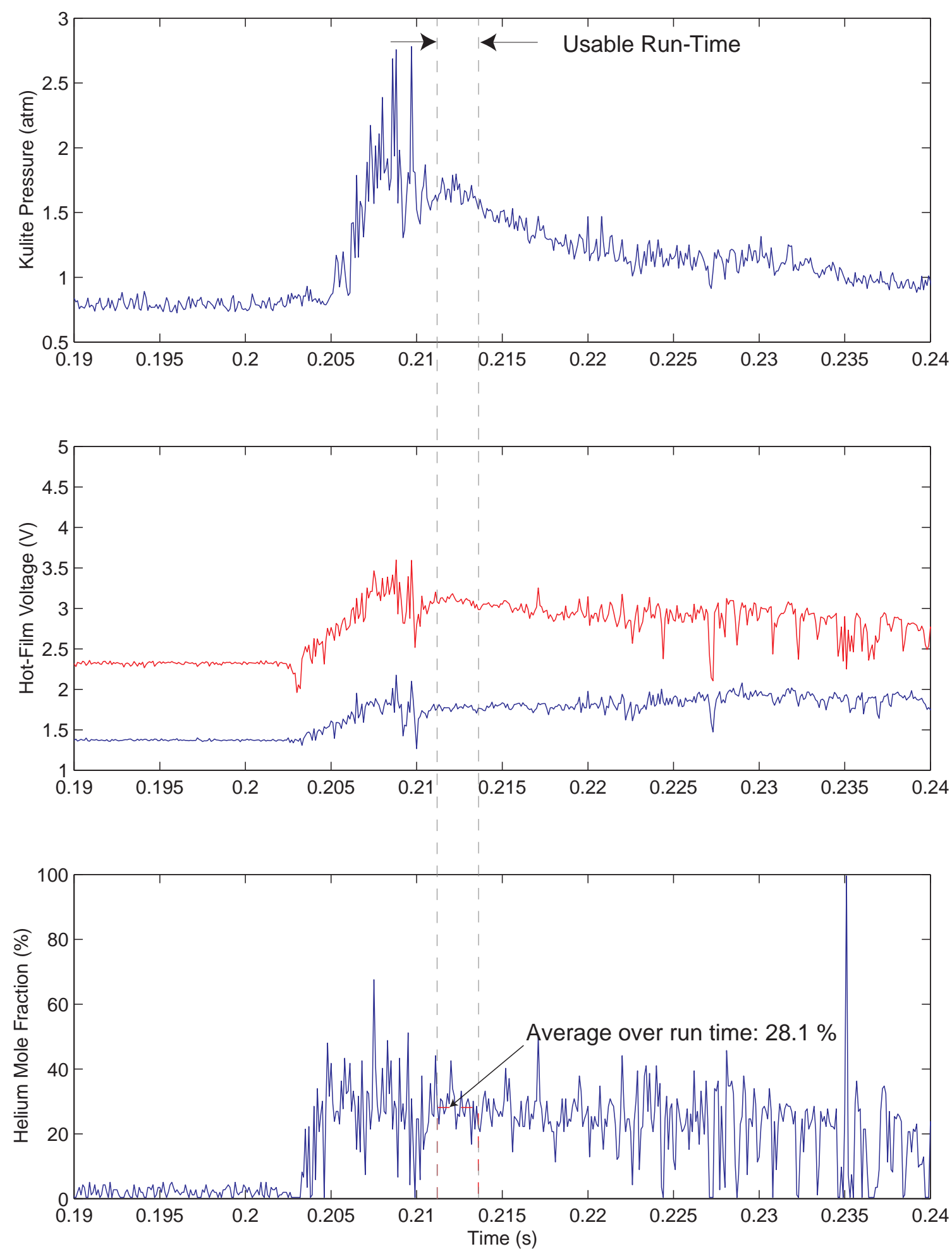

Figure 16 - Concentration: 25\% 

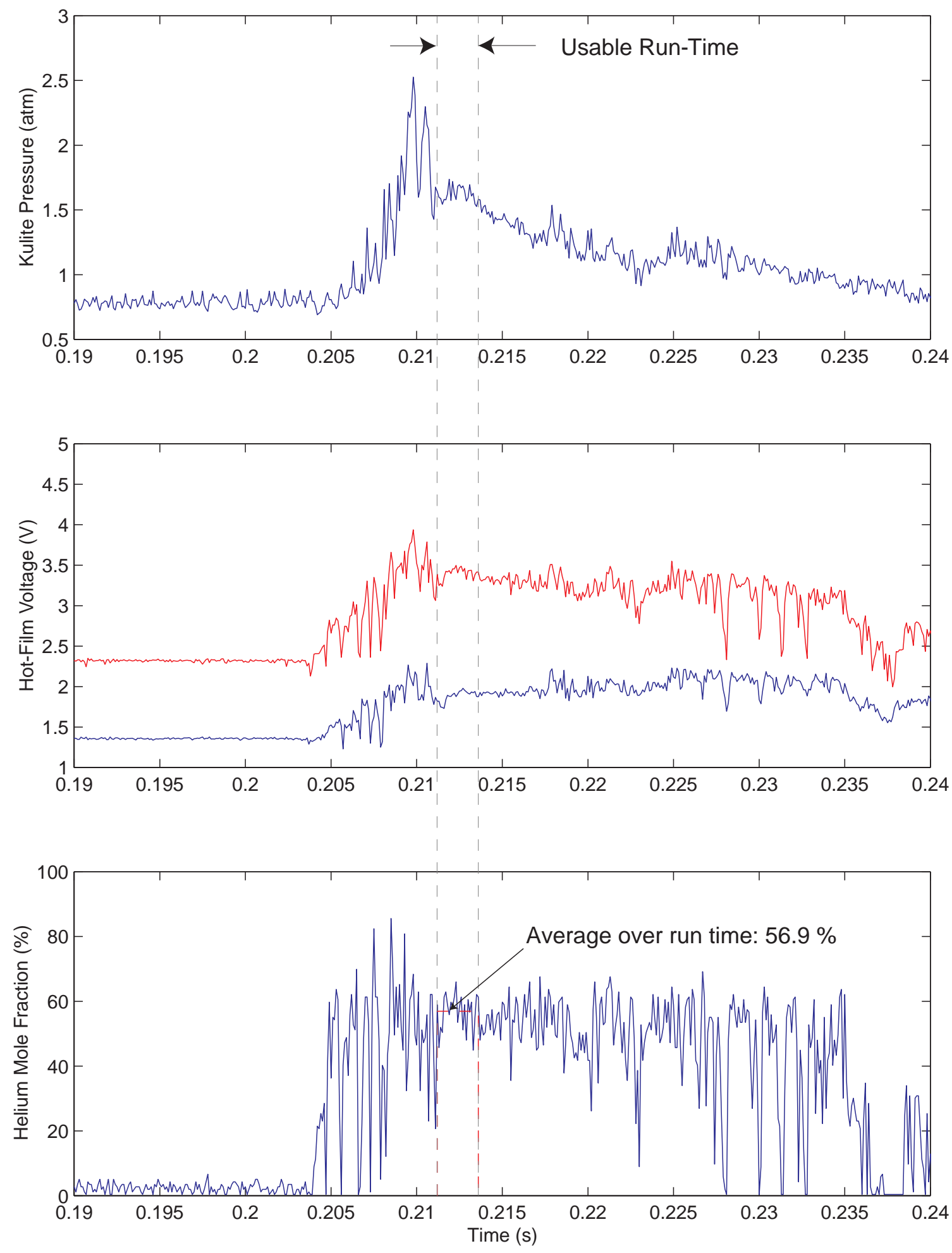

Figure 17 - Concentration: 50\% 

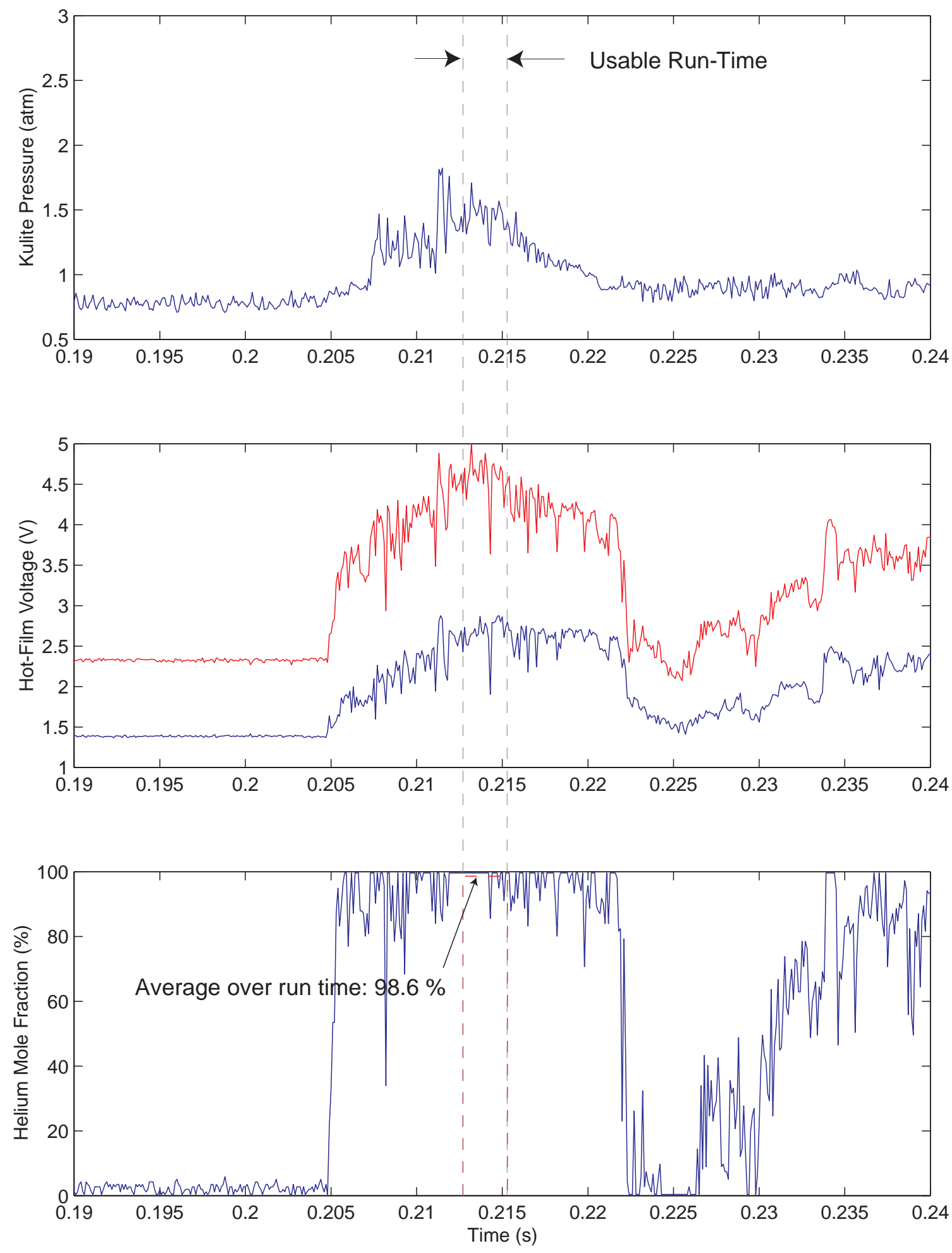

Figure 18 - Concentration: $100 \%$ 
As we can see, the results looks correct. For pure air ( $0 \%$ concentration), the resulting concentration stays near $0 \%$. However, we notice a peak at $30 \%$ when the tunnel starts. We believe it is due to unsteady effects. The helium-air cases shows a good agreement with the expected results. The $50 \%$ and $25 \%$ helium cases seem to overpredict the value of the concentration by $5 \%$ in concentration. We think it is due to the fact that we do not have the value of the constants a and $\mathrm{m}$ for this concentration level. Instead the values of $\mathrm{a}$ and $\mathrm{m}$ are interpolated from the values known at other concentration levels.

Assessing the frequency response of the probe is the final and most important test. The first idea was to create high frequency concentration fluctuations and analyze the response of the probe to these fluctuations. Unfortunately, we could not find an easy or cheap way to do it. The next idea was to put the probe through a concentration step change. The way it is usually done is to use a shock tube. However, this solution presented some majors concerns; the pressures involved are high and fragments of the diaphragm could impact on the hot films and break them. We decided to use the hypersonic wind tunnel instead. The start time is very short and can simulate a step change. Thus, we expect to see a near step change in the concentration. A typical result is shown in Figure 19.

Calling $\mathrm{t}_{0}$ the time where we first see a change in concentration, we defined an idealized theoretical concentration signal as:

$$
\begin{aligned}
& X_{\text {theo }}=\bar{X}\left(t<t_{0}\right) \\
& X_{\text {theo }}=\bar{X}\left(t \geq t_{0}\right)
\end{aligned}
$$




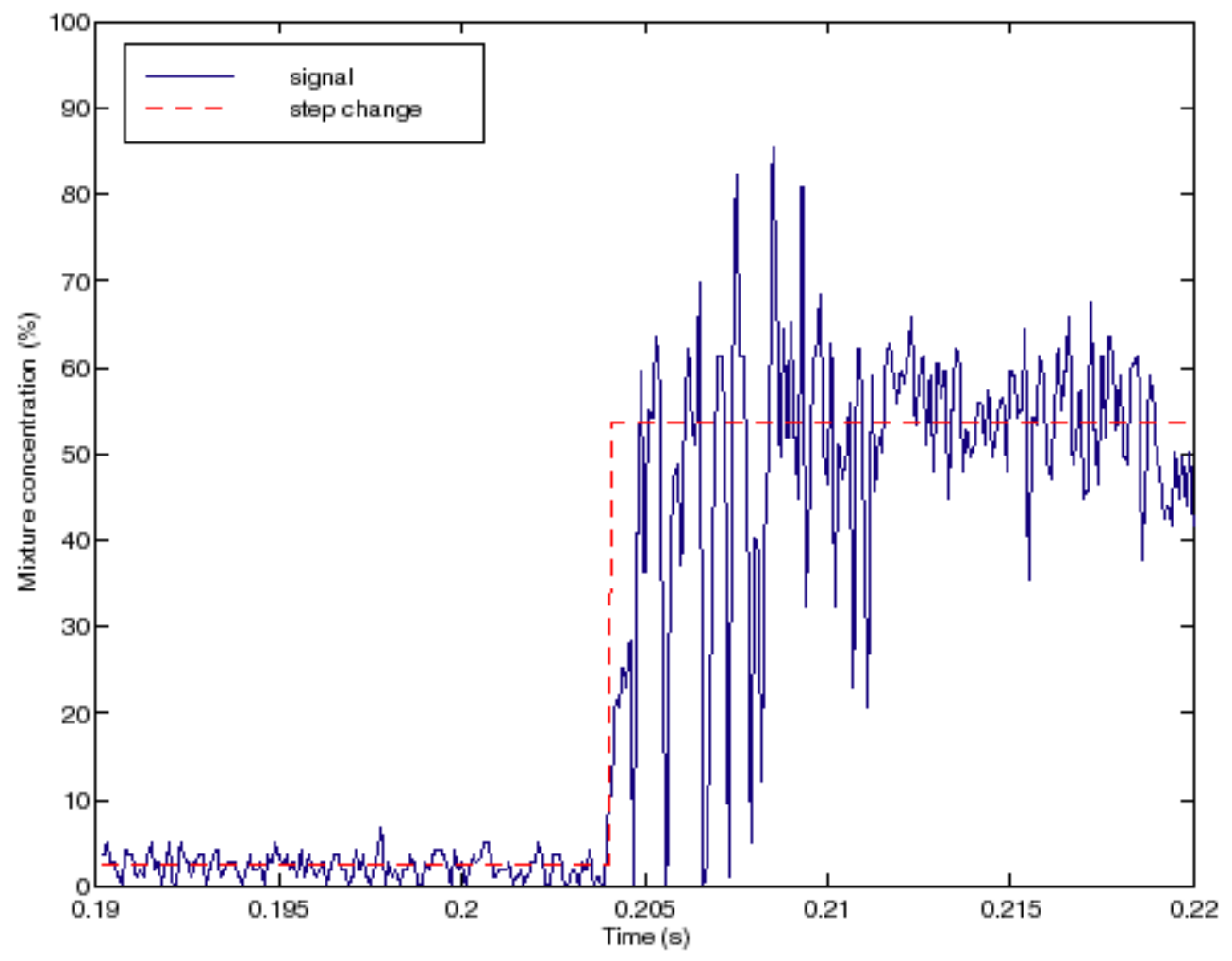

Figure 19 - Concentration signal and idealized step change

We can transform this to compute the response to an impulse signal. We can then form the actual frequency response function of the probe as:

$$
H(f)=\frac{\bar{F}(\text { impulse }) \cdot F(\text { signal })}{\bar{F}(\text { impulse }) \cdot F(\text { impulse })}
$$

(Eq. 28)

We then plotted the gain of this function (the DC component has been discarded) in Figure 20 for a typical case. We also plotted an average of all the gains obtained in Figure 21: 


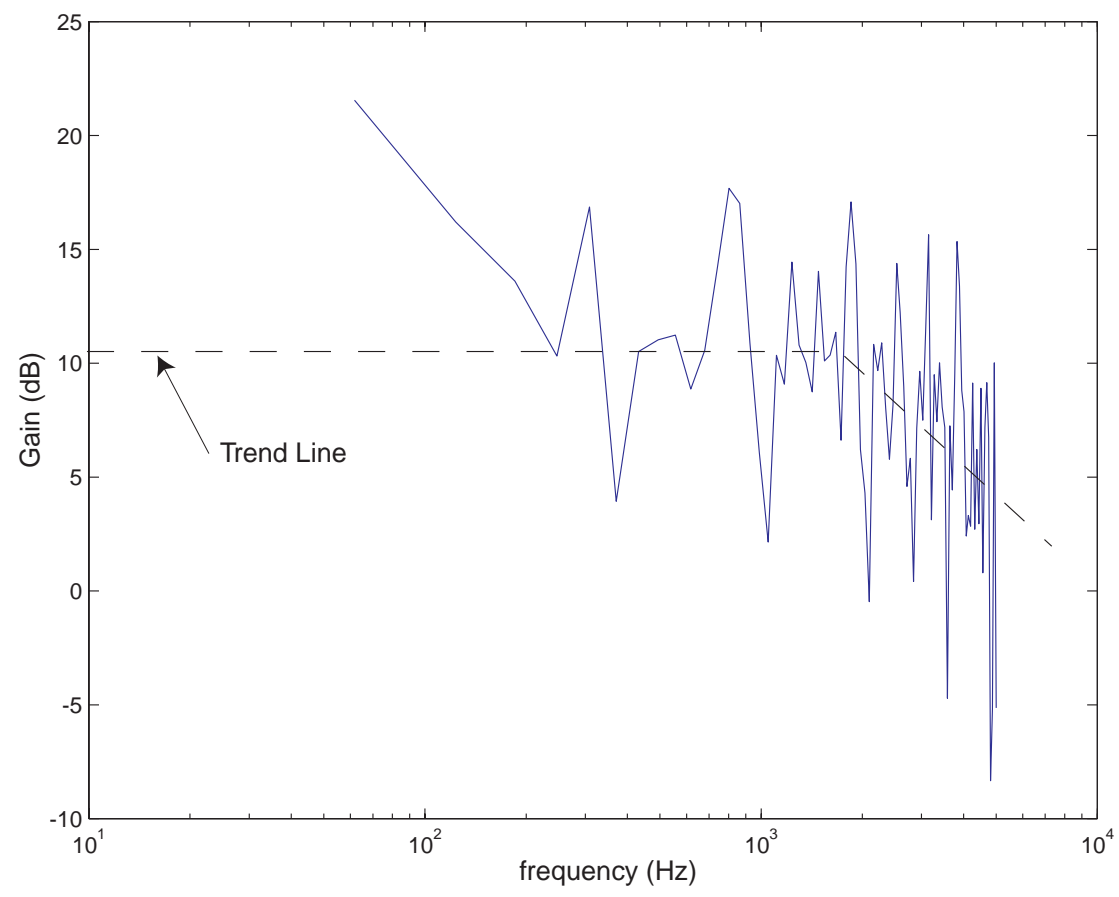

Figure 20 - Gain of the frequency response function

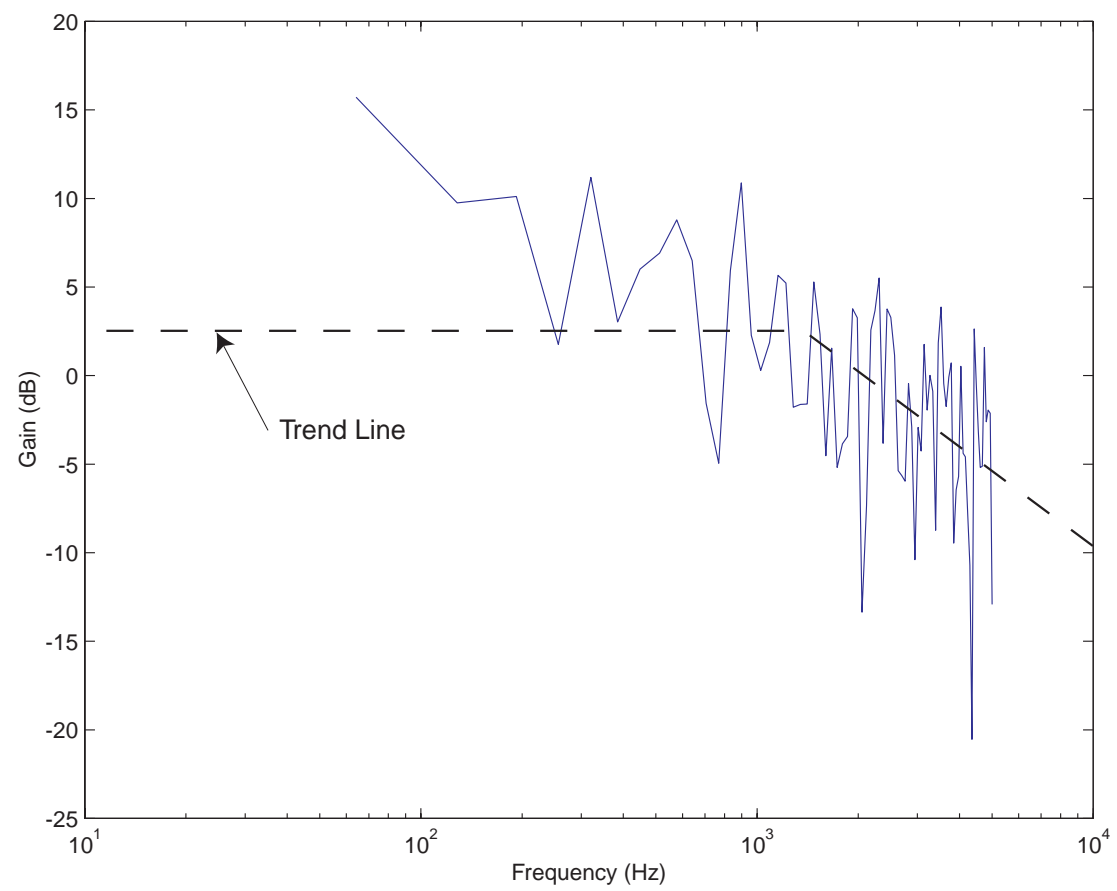

Figure 21 - Gain of the frequency response function (averaged) 
These plots show that the frequency response of the probe is about $2 \mathrm{kHz}$, which is higher than the design goal of $1 \mathrm{kHz}$. The response is flat up to about $1.5 \mathrm{kHz}$ and then begins to slowly decrease. The low frequency part of the plot is not very well defined due to the number of points we used in the Fourier Transform. By sampling at a higher rate we could get a better resolution in the low frequencies and obtain a higher frequency on the other side of the plot. 
The physical design of the probe was the most arduous part. Finding a compromise between the limiting factors and the desired features was not an easy task, and we needed several attempts before achieving final success. The sampling area is bigger than other sampling probes (0.086 in. diameter); this was imposed by the size of the hot-film probe and the necessity for them to work in the supersonic region of the probe. The choice for the hot-film probe to be placed in front of the swallowed shock proved to reduce the noise on the anemometer signals. However, the Kulite transducer presents a lot of noise. We believe this is due to the turbulence generated by the films and their supports. This noise is the main source of uncertainties in measuring the concentration. The location of the Kulite transducer also gives concerns about measuring the total pressure. One alternative solution would be to measure the static pressure at the throat.

Despite all this comments, the probe works properly and gives very good hopes about its frequency response. It seems that it can be used up to $2.5 \mathrm{kHz}$. The main limiting factor in achieving a high-frequency response is the internal volume of the probe. The necessary separation of the pressure transducer and the hot-films put a barrier on the highest achievable frequency response.

In any case, this probe demonstrated that it was possible to do high-frequency measurements of the local fluctuations of concentration of a Helium-Air mixture in a supersonic flow. The main improvements would be to increase the accuracy of the probe and to find a way to reduce the workload needed for calibration. 
D.BESTION, J. GAVIGLIO, J. P. BONNET "Comparison between constant-current and constant -temperature hot-wire anemometers in high-speed flows" Review of Scientific Instruments vol. 54, No.11, November 1983 (pp. 1513-1524)

J. M. BURGERS “Flow Equations for composite gases” Academic Press, 1969

J. -F. DEVILLERS, G. B. DIEP "Hot-wire Measurements of Gas Mixture concentrations in a Supersonic Flow" DISA INFO. 14, 1973 (pp. 29-36)

R. FULLER "Fuel-Vortex Interactions for enhanced mixing in supersonic flow" Ph. D. Thesis, Virginia Polytechnic Institute and State University, Blacksburg, 1996

F. GOBULEV “Viscosity of gases and gas mixtures - A Handbook” Israel Program for Scientific Translations, 1970

Ya. KONKIN, V. A. LEBIGA, V. N. ZINOVIEV "Determination of an aspirating probe frequency response" I.C.M.A.R'96 Novosibirsk, Russia - Proceedings Part 2

H. W. LIEPMANN, A. ROSHKO "Elements of Gasdynamics" Galcit Aeronautical Series, John Wiley \& Sons, Inc, 1957

R. J. MOFFAT "Contributions to the Theory of Single-Sample Uncertainty Analysis" Transactions of the ASME vol. 104, June 1982, pp. 250-260

M. V. MORKOVIN "Fluctuations and Hot-Wire Anemometry in Compressible Flows" AGARDograph 24, Nov. 1956 
W. F. NG, A. H. EPSTEIN "High-frequency temperature and pressure probe for unsteady compressible flows" Review of Scientific Instruments vol. 54, No.12, December 1983 (pp. 1676-1683)

T. A. NINNEMANN, W. F. NG "A concentration probe for the study of mixing in supersonic shear flows" Experiments in Fluids 13, 1992 (pp. 98-104)

A. J. SMITS, K. HAYAKAWA, K. C. MUCK "Constant Temperature Hot-Wire Anemometer Practice in Supersonic Flows - Part 1: The Normal Wire" Experiments in Fluids 1, 1983 (pp. 83-92)

J. A. SCHETZ "Boundary Layer Analysis” Prenctice Hall, 1991

"IFA 100 Intelligent Flow Analyzer Instruction Manual”, TSI Incorporated, 1983 
This appendix presents the physical properties of Air and Helium. Some properties depend on the temperature and the formulas used to evaluate them are based on a curvefit (Fuller (1996)).

This appendix also shows the relation to find the properties of the mixture of concentration $\mathrm{X}$ from the properties of Air and Helium. These relations are based on perfect gas mixtures and other relations found in the literature (Burgers (1969), Devillers and Diep (1973), Gobulev (1970), Schetz (1991)).

\section{Air properties}

Molecular weight $\quad \mathrm{M}_{\text {air }}=28.97{\mathrm{~g} . \mathrm{mol}^{-1}}^{-1}$

Gas constant

$$
R_{\text {air }}=\frac{\Re}{\mathrm{M}_{\text {air }}}=286.98
$$

Specific heat

$$
C p_{\text {air }}=\frac{\left(28110+1.967 \cdot T+4.802 \cdot 10^{-3} \cdot T^{2}-1.966 \cdot 10^{-7} \cdot T^{3}\right)}{\mathrm{M}_{\text {air }}}
$$

Ratio of specific heats $\gamma_{\text {air }}=\frac{C p_{\text {air }}}{C p_{\text {air }}-R_{\text {air }}}$

Viscosity

$$
\begin{aligned}
\mu_{\text {air }} & =-1.31554 \cdot 10^{-6}+9.53265 \cdot 10^{-8} \cdot T-1.50660 \cdot 10^{-10} \cdot T^{2} \\
& +2.41737 \cdot 10^{-13} \cdot T^{3}-2.58576 \cdot 10^{-16} \cdot T^{4}+1.26849 \cdot 10^{-19} \cdot T^{5} \\
k_{\text {air }} & =1.61344 \cdot 10^{-4}+8.89970 \cdot 10^{-5} \cdot T+3.85599 \cdot 10^{-8} \cdot T^{2} \\
& -2.39332 \cdot 10^{-10} \cdot T^{3}+3.48891 \cdot 10^{-13} \cdot T^{4}-1.84858 \cdot 10^{-16} \cdot T^{5}
\end{aligned}
$$

Conductivity

\section{Helium properties}

Molecular weight $\quad \mathrm{M}_{\mathrm{He}}=4.0026{\mathrm{~g} . \mathrm{mol}^{-1}}^{-1}$ 
Gas constant

$$
R_{H e}=\frac{\Re}{\mathrm{M}_{H e}}=2077.15
$$

Specific heat

$$
C p_{H e}=5192.6
$$

Ratio of specific heats $\gamma_{\mathrm{He}}=\frac{C p_{\mathrm{He}}}{C p_{\mathrm{He}}-R_{\mathrm{He}}}=1.6667$

Viscosity

$$
\begin{aligned}
\mu_{H e} & =-4.56080 \cdot 10^{-6}+2.05152 \cdot 10^{-7} \cdot T-8.89707 \cdot 10^{-10} \cdot T^{2} \\
& +2.41714 \cdot 10^{-12} \cdot T^{3}-3.20720 \cdot 10^{-16} \cdot T^{4}+1.63060 \cdot 10^{-18} \cdot T^{5} \\
k_{H e} & =4.33185 \cdot 10^{-2}+1.23854 \cdot 10^{-4} \cdot T+2.77149 \cdot 10^{-6} \cdot T^{2} \\
& -1.11774 \cdot 10^{-8} \cdot T^{3}+1.81601 \cdot 10^{-11} \cdot T^{4}-1.03892 \cdot 10^{-14} \cdot T^{5}
\end{aligned}
$$

Conductivity

\section{Perfect Gases mixture relations}

Molecular weight $\quad \mathrm{M}=(1-X) \mathbf{M}_{\text {air }}+X \mathrm{M}_{H e}$

Gas constant $\quad R=\frac{\Re}{\mathrm{M}}$

Specific heat $\quad C p=C p_{\text {air }}+C p_{H e}$

Ratio of specific heats $\gamma=\frac{C p}{C p-R}$

Viscosity

$$
k=\frac{1}{2}\left((1-X) k_{\text {air }}+X k_{H e}+\frac{k_{\text {air }} \cdot k_{H e}}{\left((1-X) \sqrt{k_{\text {air }}}+X \sqrt{k_{H e}}\right)^{2}}\right)
$$

Conductivity

$$
\mu=\frac{\mu_{\text {air }}}{1+\zeta_{\text {air } H e}\left[\frac{1-X}{X}\right]}+\frac{\mu_{H e}}{1+\zeta_{\text {Heair }}\left[\frac{X}{1-X}\right]}
$$

where $\zeta_{\text {air } H e}=\frac{\left[1+\left(\frac{\mathbf{M}_{H e}}{\mathbf{M}_{\text {air }}}\right)^{\frac{1}{4}} \sqrt{\frac{\mu_{\text {air }}}{\mu_{H e}}}\right]}{\frac{4}{\sqrt{2}} \sqrt{1+\frac{\mathbf{M}_{\text {air }}}{\mathbf{M}_{H e}}} \text { and } \zeta_{\text {Heair }}}=\frac{\left[1+\left(\frac{\mathbf{M}_{\text {air }}}{\mathbf{M}_{H e}}\right)^{\frac{1}{4}} \sqrt{\frac{\mu_{H e}}{\mu_{\text {air }}}}\right]}{\frac{4}{\sqrt{2} \sqrt{1+\frac{\mathbf{M}_{H e}}{\mathbf{M}_{\text {air }}}}}}$ 
This appendix presents the computer codes used. The programs are written in $\mathrm{C}++$. Two main programs are described. The calibration code was used to find the calibration constants from the experiments in the calibration tank. The reduction code is the one used to reduce a particular set of data in order to find the concentration. Both these codes use two modules. The first one implements the calculation of the properties of the mixture. The second one is used to read the configuration of the probe and of the transducers.

\section{Calibration Code}

This program is used to determine the calibration constants. It takes sets of data files for every concentration level and return a file (calibr8.dat) containing the calibration constants for every concentration level. The input data files are listed in the file calfiles.lst where they are sorted by concentration level. Each data file is an ascii file composed of 5 columns, respectively time, hot-film voltage, hot-film voltage, Kulite pressure and backpressure. The fifth column is not used but is required even if it is filled with zeros. The geometry and characteristics of the probe are given in the file probe.dat which is read at the beginning of the program.

For every concentration level, we proceed to a least square curve-fit using all the data points of the files.

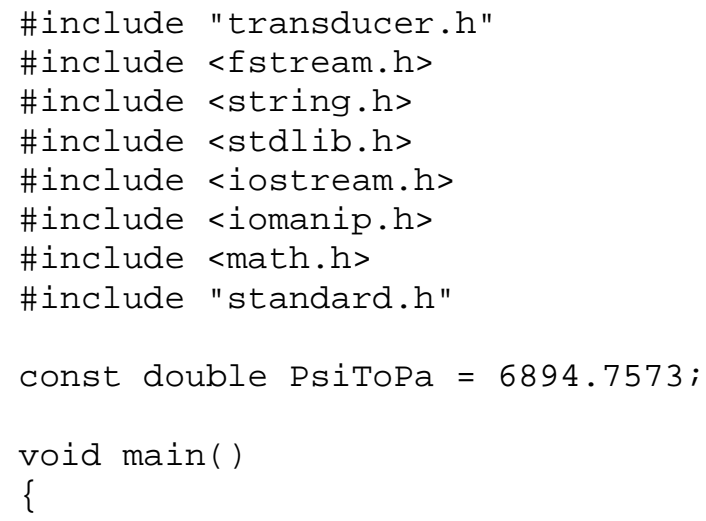


cout $<<"$

$"<<$

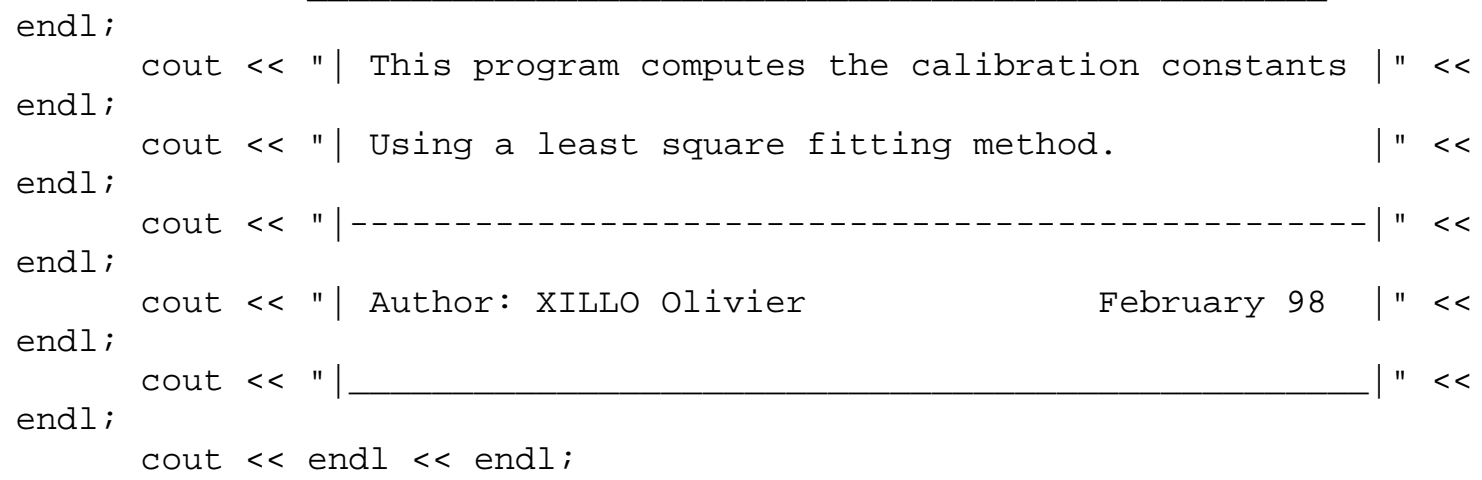




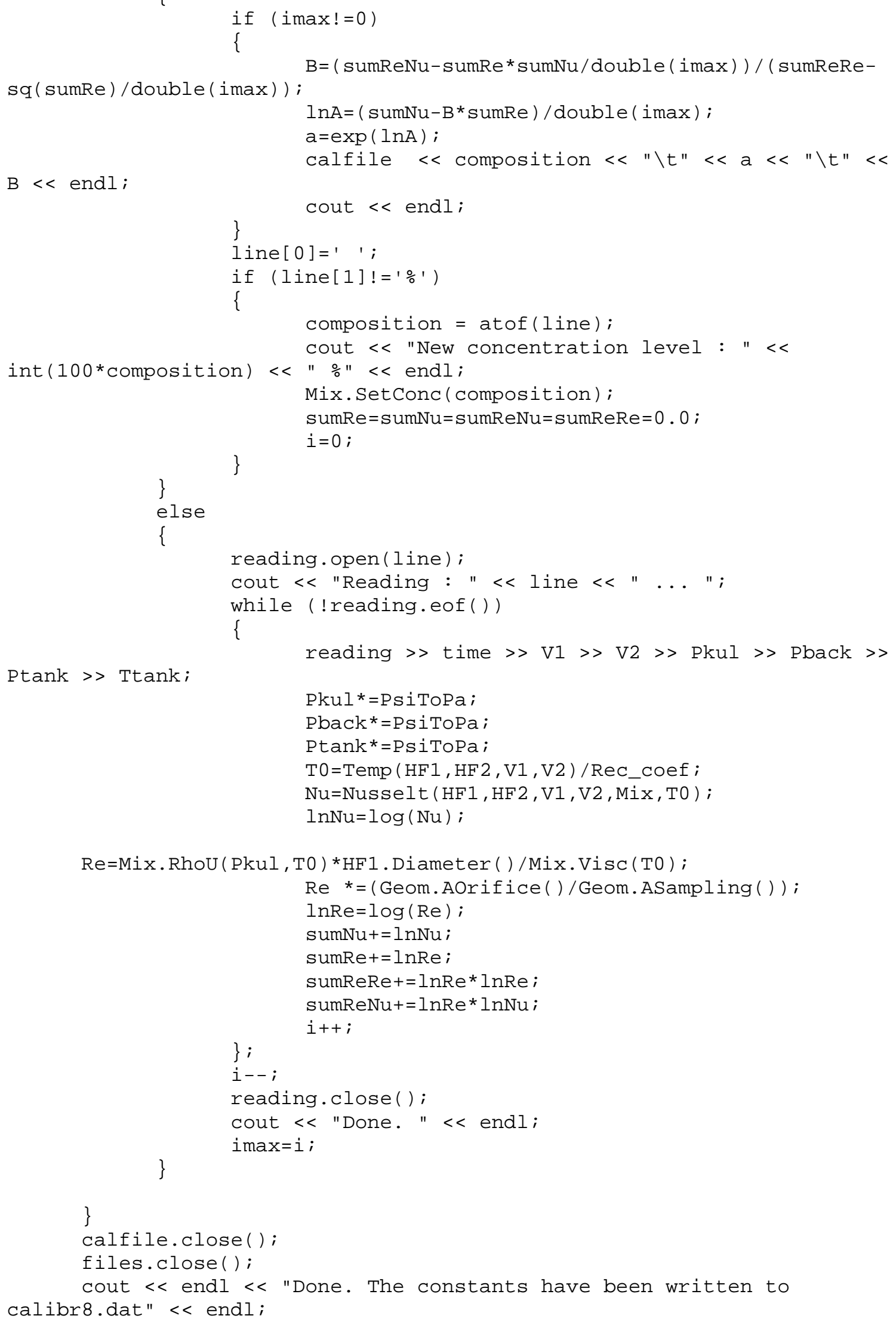


cout $<$ endl $<$ "Press any key to exit ..." $<$ endl;

$\operatorname{getch}() ;$

\}

\section{Data Reduction Code}

This program is used to reduce the data. It takes one data file at a time and return an ascii file with the 5 followings columns: time, hot-film voltage, hot-film voltage, Kulite pressure in $\mathrm{Pa}$ and concentration. To determine the concentration, the program uses a dichotomy method. The calibration constants a and $\mathrm{m}$ are read from the file calibr8.dat. If the constants do not exist for a given concentration, they are linearly interpolated. Finally, geometry and characteristics of the probe are read from the file probe .dat.

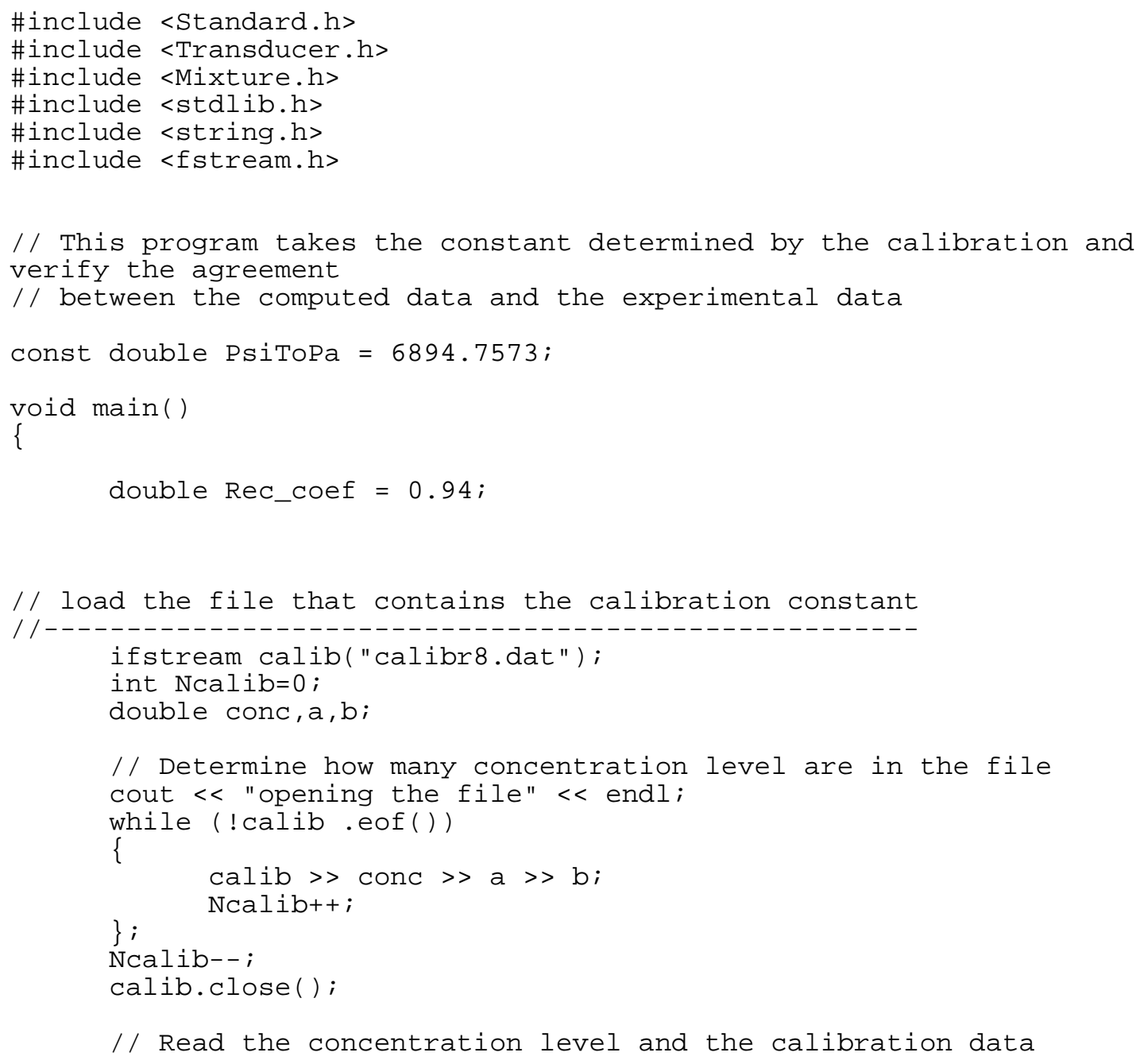




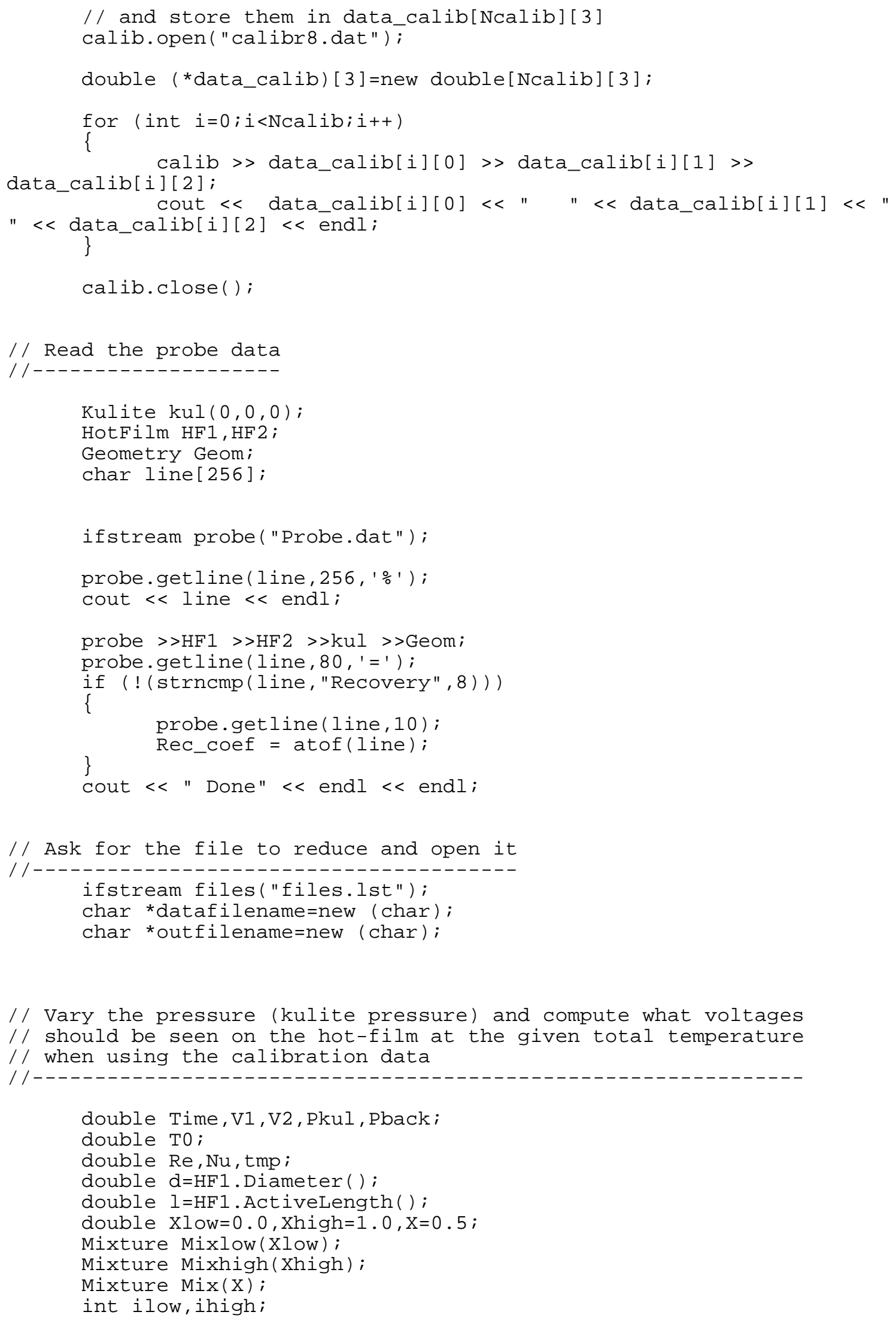




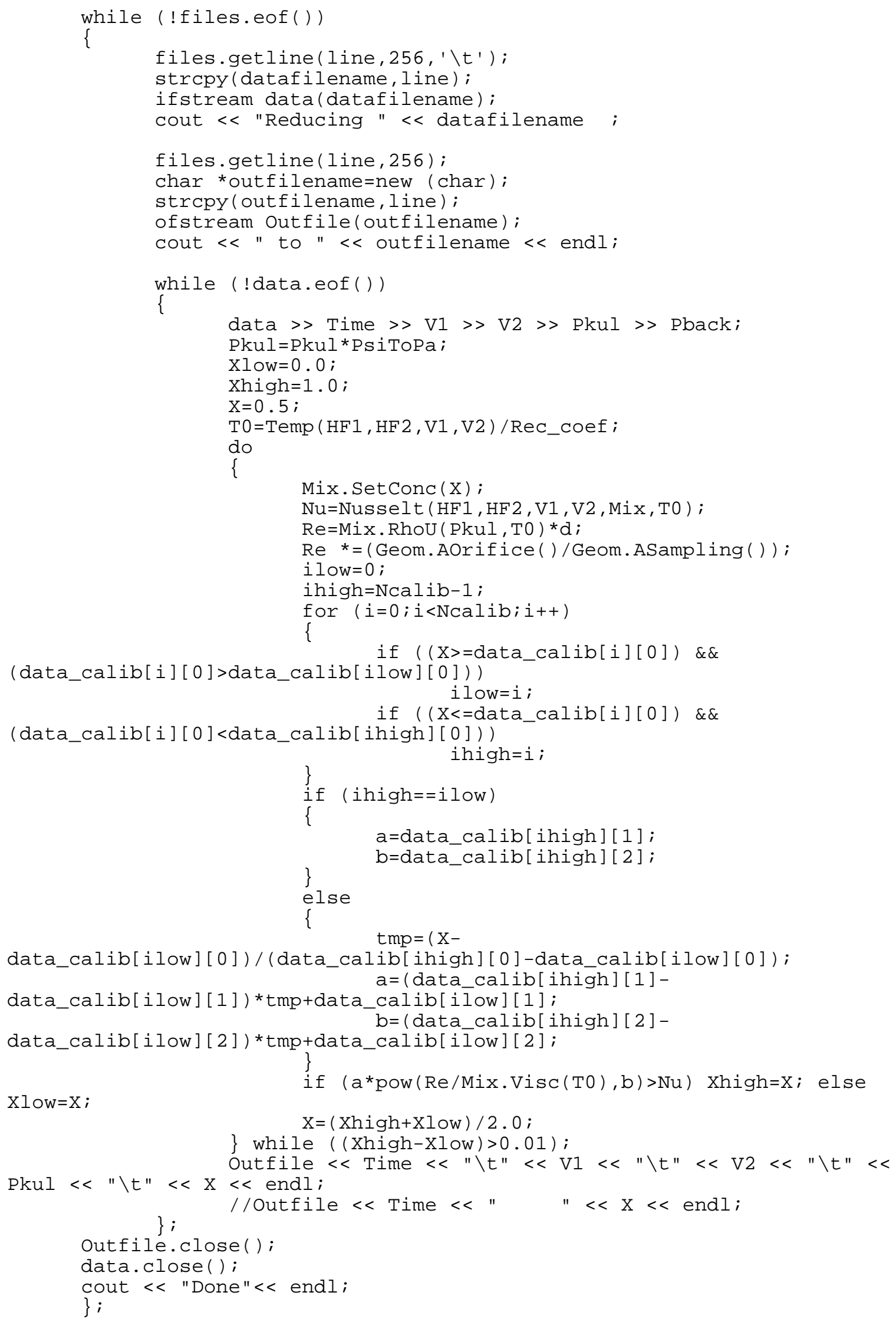


files.close ();

cout $<$ endl $<<$ "Press any key to exit..." $<$ endl; getch ( ) ;

\} 


\section{Common components}

The two followings code are used by both the calibration program and the data reduction program. The code mixture.cpp presents functions to compute the properties of gas mixtures. The code transducer.cpp implements functions to read the properties of the probe and of the transducers used. It also implements routines to compute the Nusselt number and temperature from the hot-film voltages.

Finally, the file probe.dat is presented with the values used.

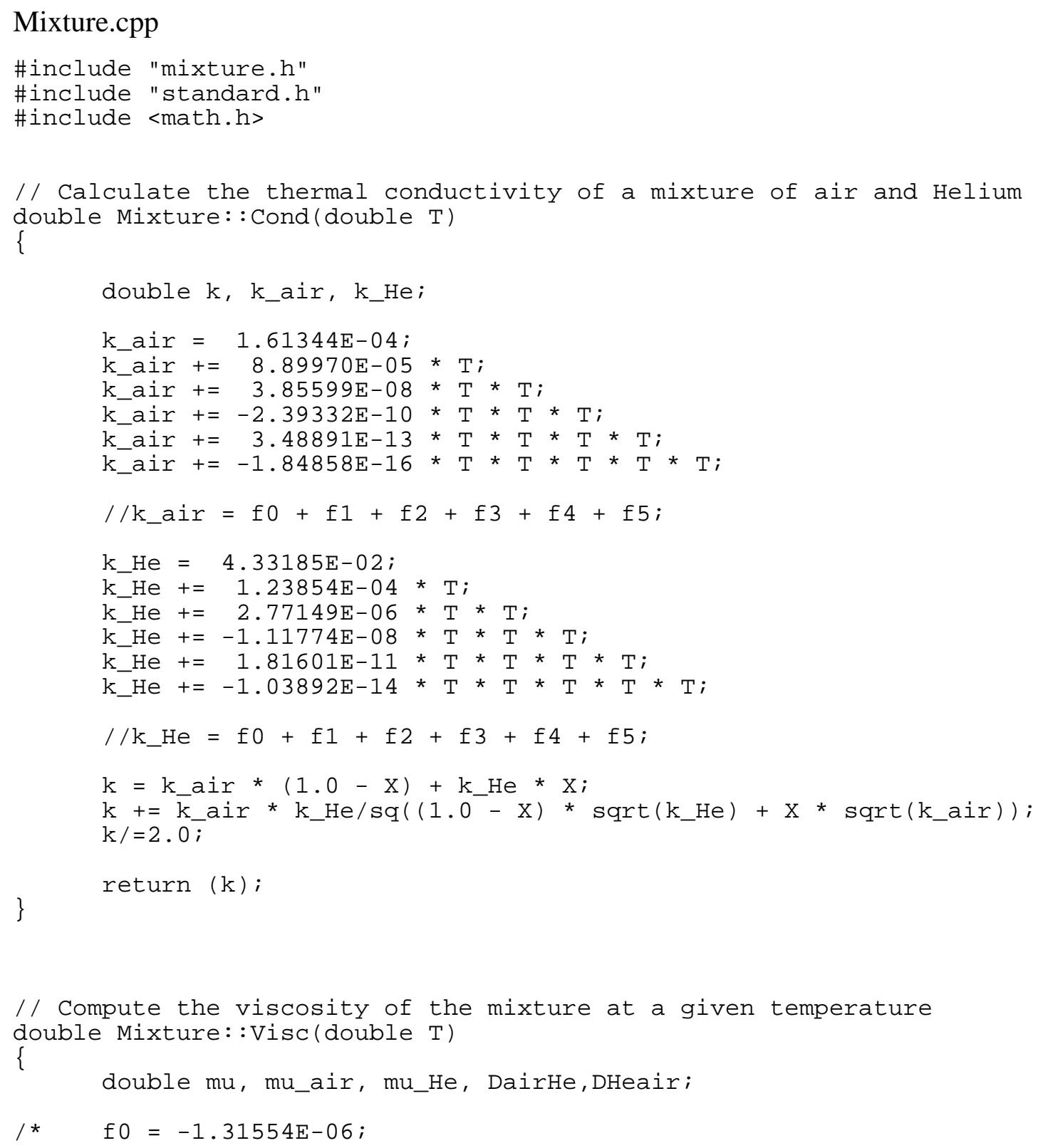




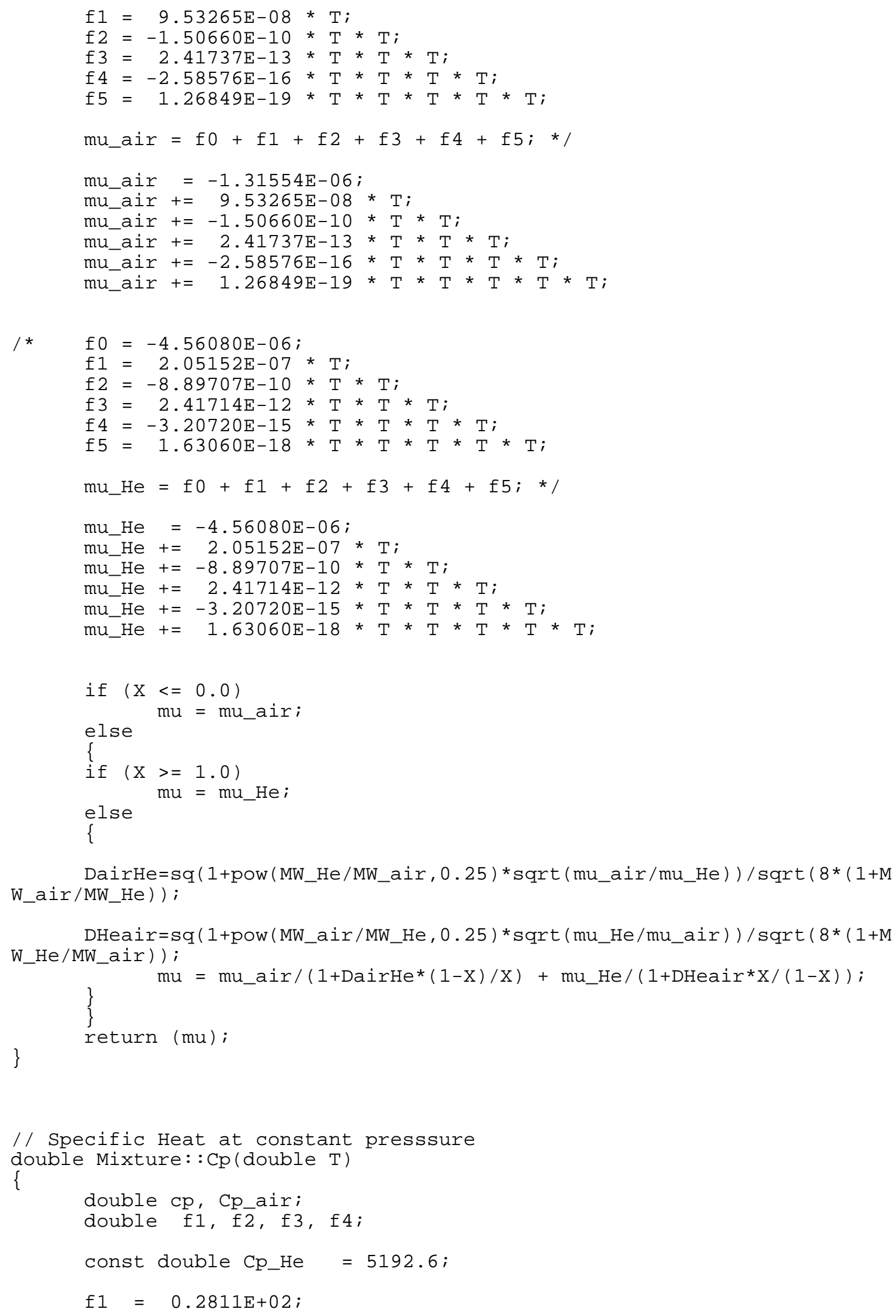




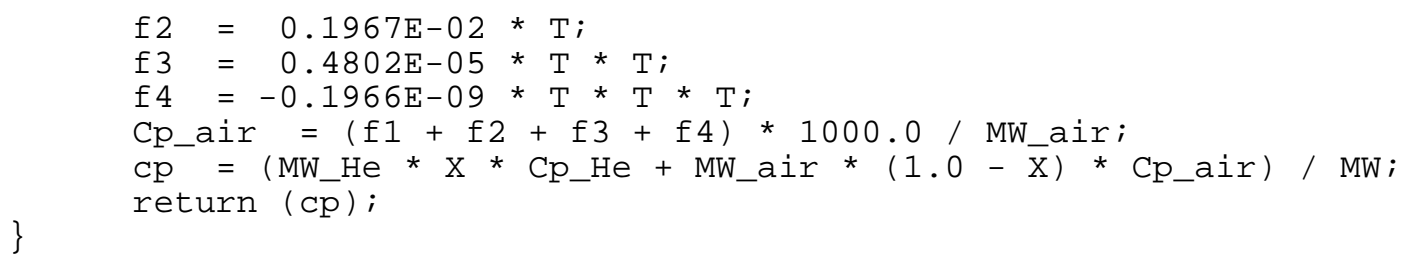




\section{Transducer.cpp}

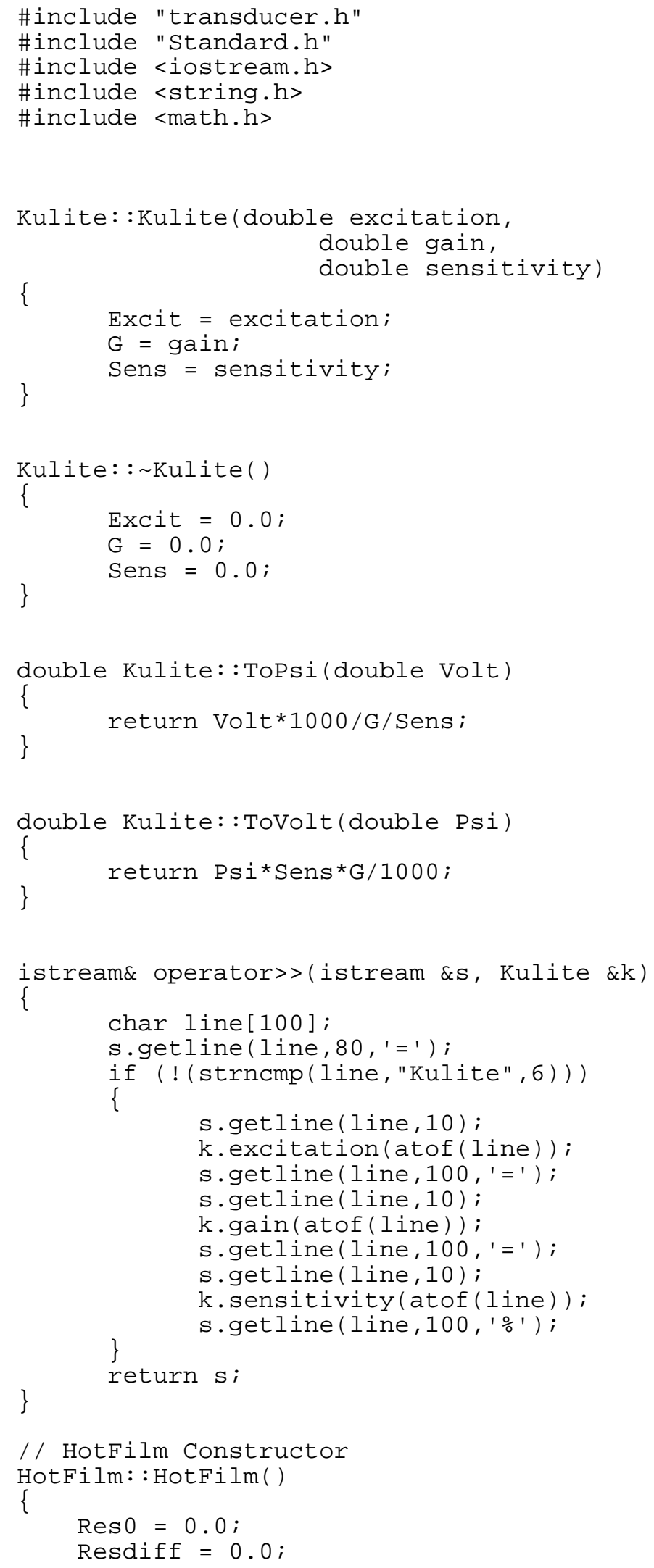




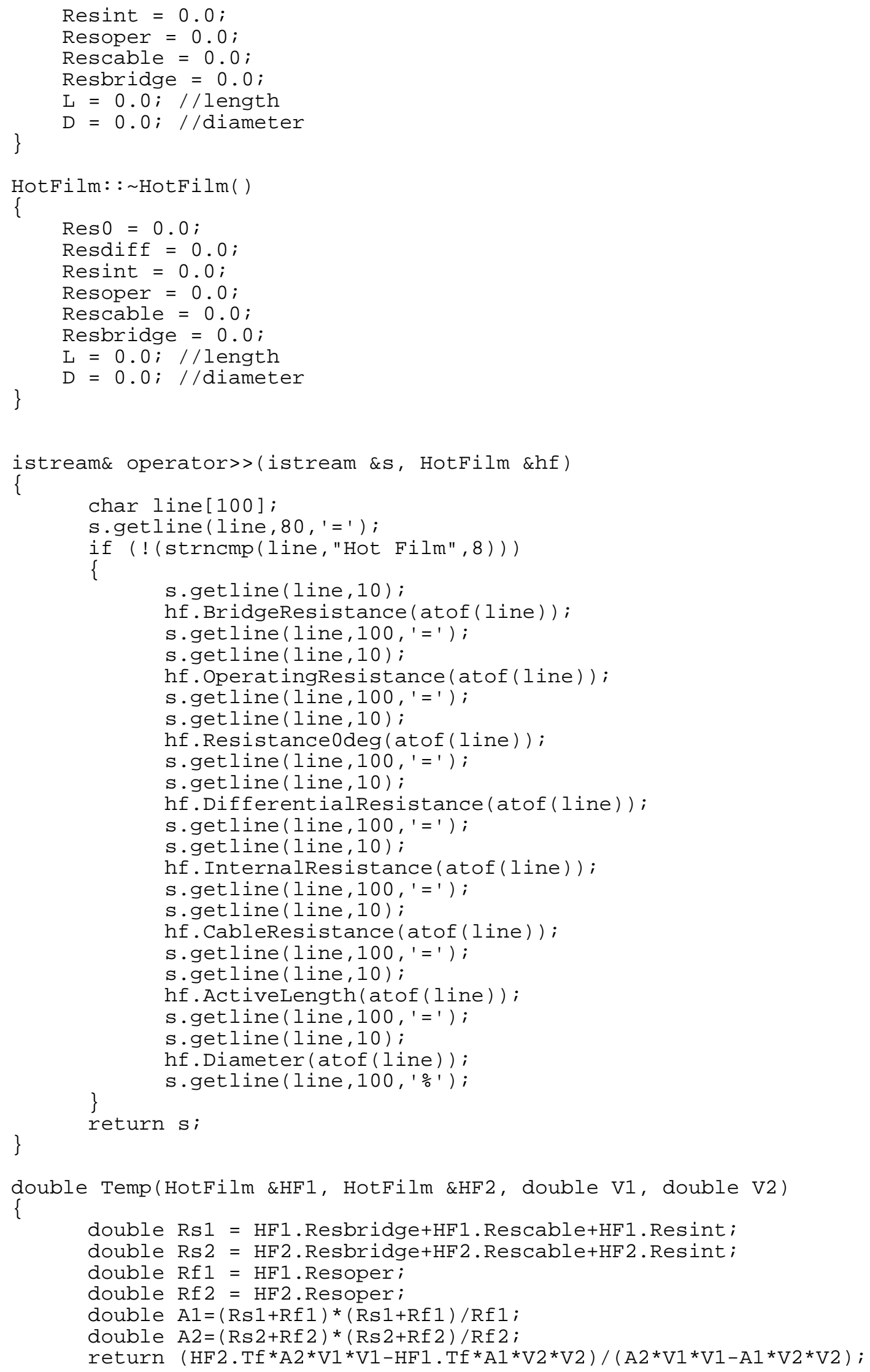


/*double TempTot (Hotfilm \&HF1, HotFilm \&HF2, double V1, double V2, double Tcoef, double EqCoef)

\{

double Rs1 = HF1.Resbridge+HF1.Rescable+HF1.Resint;

double Rs2 = HF2. Resbridge+HF2. Rescable+HF2. Resint;

double $\operatorname{Rf} 1=H F 1$. Resoper;

double Rf2 = HF2. Resoper;

double $A 1=(\operatorname{Rs} 1+\operatorname{Rf} 1)$ * $(\operatorname{Rs} 1+\operatorname{Rf} 1) / \operatorname{Rf} 1$;

double $A 2=(\operatorname{Rs} 2+\operatorname{Rf} 2)$ * $(\operatorname{Rs} 2+\operatorname{Rf} 2) / \operatorname{Rf} 2$;

double a $1=\mathrm{V} 1 * \mathrm{~V} 1 / \mathrm{A} 1 / \mathrm{HF} 1 . \mathrm{L}$;

double a $2=\mathrm{V} 2 * \mathrm{~V} 2 / \mathrm{A} 2 / \mathrm{HF} 2 . \mathrm{L}$;

double $a=(a 1-a 2) *(E q C o e f+T$ Coef $*$ EqCoef*EqCoef $)$;

double $\mathrm{b}=(1.0+2.0 *$ Tcoef*EqCoef $) *(\mathrm{a} 2 * \mathrm{HF} 1 . \mathrm{Tf}-\mathrm{a} 1 * \mathrm{HF} 2 . \mathrm{Tf})$;

double $\mathrm{C}=\mathrm{Tcoef} *\left(\mathrm{a} 1{ }^{*} \mathrm{HF} 2 . \mathrm{Tf}{ }^{*} \mathrm{HF} 2 . \mathrm{Tf}-\mathrm{a} 2{ }^{*} \mathrm{HF} 1 . \mathrm{Tf}{ }^{*} \mathrm{HF} 1 . \mathrm{Tf}\right)$;

double delt $\mathrm{a}=\mathrm{b} * \mathrm{~b}-4{ }^{*} \mathrm{a}{ }^{*} \mathrm{c}$;

\}$* 1$

return (-b+sqrt (delta)) /2.0/a;

double Nusselt(HotFilm \&HF1, HotFilm \&HF2, double V1, double V2, Mixture $\& M$, double Temp)

\{

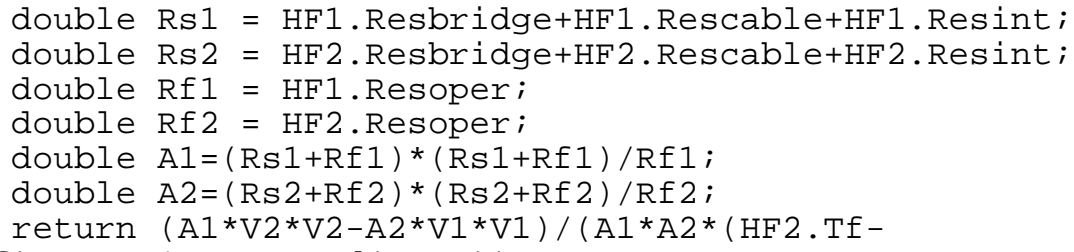




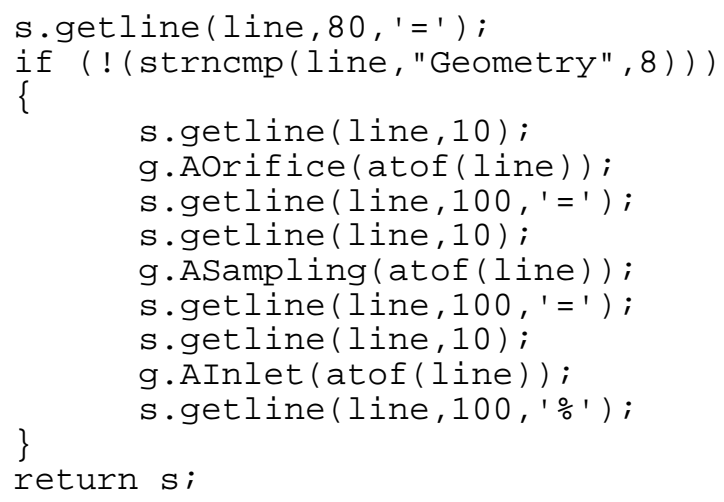




\section{Probe.dat}

\%Hot Film 1

Anemometer bridge resistance
Sensor operating resistance

Sensor resistance a 0 deg C

Res @ 100 deg - Res @ 0 deg

Internal Resistance

Added cable resistance

Active length of hot-film

Diameter of hot-film

$(\mathrm{Ohm})=10$

$(\mathrm{Ohm})=8.208$

$(\mathrm{Ohm})=4.94$

$(\mathrm{Ohm})=1.20$

$(\mathrm{Ohm})=0.46$

$(\mathrm{Ohm})=0.593$

(m) $=0.51 \mathrm{e}-3$

(m) $\quad=25.4 e-6$

\%Hot Film 2

Anemometer bridge resistance

Sensor operating resistance

Sensor resistance a 0 deg C

Res a 100 deg - Res a 0 deg

Internal Resistance

Added cable resistance

Active length of hot-film

Diameter of hot-film

$(\mathrm{Ohm})=10$

$(\mathrm{Ohm})=6.112$

$(\mathrm{Ohm})=4.90$

$(\mathrm{Ohm})=1.19$

$(\mathrm{Ohm})=0.49$

$(\mathrm{Ohm})=0.595$

$(\mathrm{m}) \quad=0.51 \mathrm{e}-3$

(m) $\quad=25.4 e-6$

oKulite

Excitation

Gain

Sensitivity

$(\mathrm{V}) \quad=5$

$=100$

$(\mathrm{mV} / \mathrm{Psi})=2.253$

\%Geometry

Area of choked orifice

$\left(m^{\wedge} 2\right)=7.405 e-6$

Area of sampling plane

$\left(\mathrm{m}^{\wedge} 2\right)=6.701 \mathrm{e}-6$

Area of inlet orifice

$\left(\mathrm{m}^{\wedge} 2\right)=3.746 \mathrm{e}-6$

\%Recovery Factor

Recovery Factor of the probe

$=0.94$

$\div$ 
An analysis of the accuracy of the probe is presented here. A truly rigorous uncertainty analysis would have required a enormous amount of work. However, a simpler technique can be used to get an estimate of the accuracy of the probe. This technique is based on the jitter program (R. J. MOFFAT (1982)). Using previously collected data points and introducing small perturbations in these data, one can determine the sensitivity of the desired quantity to changes in the measured quantities. In our case, we can determine the sensitivity of the concentration to changes in the pressure from the Kulite pressure transducer or in the voltage from the hot-films. Writing $X=g\left(V_{1}, V_{2}, P_{k u l}\right)$, we are evaluating the partial derivative $\frac{\partial g}{\partial V_{1}}, \frac{\partial g}{\partial V_{2}}$ and $\frac{\partial g}{\partial P_{k u l}}$. The error can then be evaluated by:

$$
\Delta X=\sqrt{\left(\frac{\partial g}{\partial V_{1}} \delta V_{1}\right)^{2}+\left(\frac{\partial g}{\partial V_{2}} \delta V_{2}\right)^{2}+\left(\frac{\partial g}{\partial P_{k u l}} \delta P_{k u l}\right)^{2}}
$$

In order to evaluate the partial derivative, we take data points from the calibration process and compute the concentration for each of them. We then perturb one of the measured quantities (e.g. pressure) and recompute the concentration. The difference in concentration divided by the perturbation gives the sensitivity coefficient. Observing the coefficient obtained, we find they vary significantly especially with the pressure. However, we can distinguish two regions. The first one is when the pressure from the Kulite transducer is low ( $<10 \mathrm{psi})$. In this region the sensitivity coefficients are big and we can expect a big error. The second region is for moderate pressures (>10 psi). In this region, the coefficients seem to diminish slowly with the pressure and we can expect a lower error. 


\section{Low pressure regime}

For all the data points with a low pressure (<10 psi), we compute the sensitivity coefficient to changes in the hot-films voltages and in the Kulite pressure. We then average them together. The resulting coefficients are:

$$
\begin{gathered}
\frac{\partial g}{\partial V_{1}}=140 \% / \mathrm{V} \\
\frac{\partial g}{\partial V_{2}}=-80 \% / \mathrm{V} \\
\frac{\partial g}{\partial P_{k u l}}=-14 \% / \mathrm{psi}
\end{gathered}
$$

Using the $95 \%$ confidence bounds for the error, we find that:

$$
\begin{gathered}
\delta V_{1}=0.073 \mathrm{~V} \\
\delta V_{2}=0.052 \mathrm{~V} \\
\delta P_{k u l}=1.734 \mathrm{psi}
\end{gathered}
$$

and the error on the concentration is $\Delta X= \pm 26.7 \%$

\section{Moderate pressure regime}

The same procedure is followed for this region. We find that:

$$
\begin{gathered}
\frac{\partial g}{\partial V_{1}}=90 \% / \mathrm{V} \\
\frac{\partial g}{\partial V_{2}}=-75 \% / \mathrm{V} \\
\frac{\partial g}{\partial P_{k u l}}=-5 \% / \mathrm{psi}
\end{gathered}
$$

and that the resulting error is $\Delta X= \pm 11.6 \%$

The main source of errors comes from the measurement of the pressure. The error does not come from the measuring device but from flow induced noise. In order to obtain a better accuracy of this probe one should consider trying to reduce this noise. 
Olivier Christian Xillo was born September 1, 1972 in Toulouse, France. After attending the Lycée Pierre de Fermat in Toulouse and obtaining his Baccalauréat in 1989, he entered the Classes Préparatoires to prepare the competitive exams to Grandes Ecoles. $\mathrm{He}$ was admitted in ENSICA (Ecole Nationale d'Ingénieurs de Constructions Aéronautiques) in 1993. He then came to Virginia Tech in 1996 to work on his end-of-study project. He returned in France to defend his thesis and got his engineer diploma in September 1996. He came back at Virginia Tech to begin his masters degree. At the same time, he began his military service as a civilian. Upon completion of his thesis in April 1998, Olivier received his master degree in Aerospace Engineering and went back to France in order to look for a job. 